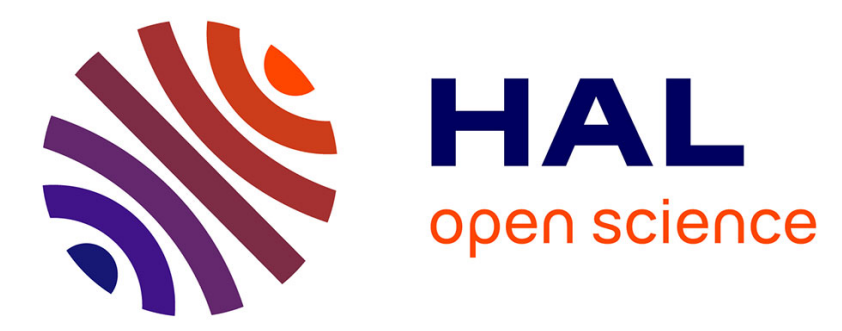

\title{
Concilier qualité nutritionnelle et qualité sensorielle des aliments
}

\author{
Elisabeth Guichard
}

\section{To cite this version:}

Elisabeth Guichard. Concilier qualité nutritionnelle et qualité sensorielle des aliments. L'alimentation à découvert, Partie II - Fabriquer les aliments : du champ à l'assiette, chapitre 8, CNRS Editions, 328 p., 2015, A découvert, 978-2-271-08300-5. hal-01249025

\section{HAL Id: hal-01249025 \\ https://hal.science/hal-01249025}

Submitted on 5 Jun 2020

HAL is a multi-disciplinary open access archive for the deposit and dissemination of scientific research documents, whether they are published or not. The documents may come from teaching and research institutions in France or abroad, or from public or private research centers.
L'archive ouverte pluridisciplinaire HAL, est destinée au dépôt et à la diffusion de documents scientifiques de niveau recherche, publiés ou non, émanant des établissements d'enseignement et de recherche français ou étrangers, des laboratoires publics ou privés. 
- DEUXIËME PARTIE -

Fabriquer

les aliments :

du champ

à l'assiette 


\section{Page précédente:}

Hungry Planet - Familles du monde. La famille Aboubakar (Darfour, Soudan), pose avec sa consommation alimentaire hebdomadaire. Budget: 1,23 \$. Photo Peter Menzel / Cosmos. 


\title{
2.1. Lintroduction de nouvelles plantes alimentaires en Europe: un panorama de l'Antiquité à nos jours
}

\author{
Michel Chauvet
}

L'essentiel de notre alimentation repose sur des espèces qui viennent à l'origine d'ailleurs. La ratatouille, plat emblématique de la cuisine méditerranéenne, contient de la tomate, du poivron et de la courgette, qui viennent d'Amérique; l'aubergine vient de l'Inde; l'oignon et l'ail, connus depuis bien plus longtemps, viennent d'Asie centrale, et l'huile d'olive de l'Asie de l'Ouest; il n'y a guère que le persil qui soit européen, car il vient probablement de Grèce.

La gamme de nos aliments s'est donc constituée au cours des siècles et des millénaires, par vagues successives liées à l'histoire des sociétés humaines. Ces aliments ont pu être produits sur place après l'introduction des plantes, ou bien importés par le commerce.

\section{Préhistoire et débuts de l'agriculture}

L'alimentation des peuples de la préhistoire est difficile à reconstituer, et devait différer d'un milieu à l'autre. Elle reposait sur la cueillette d'un très grand nombre de végétaux, qui apportaient les trois grandes classes de nutriments indispensables, les glucides (graines, fruits, tubercules, glands, écorces...), les protéines (graines encore, mais aussi feuilles) et les lipides (faînes, noisettes...). Les hommes ont dû très tôt inventer des procédés élaborés pour ôter à certains aliments leur toxicité et les conserver.

L'invention de l'agriculture a constitué le premier changement fondamental. Dans le Croissant fertile, il y a environ 10000 ans, tout un cortège de «plantes fondatrices » a été domestiqué, à savoir deux blés (engrain et amidonnier), l'orge, la lentille, le pois, le pois-chiche et le lin. Lors de la diffusion de l'agriculture dans d'autres régions, de nouvelles plantes s'y sont adjointes, comme la fève ou les lupins, commençant parfois leur évolution en tant que mauvaises herbes, comme le seigle ou l'avoine. Le blé tendre, quant à lui, n'existe pas dans la nature et est né vers le sud de la Caspienne par hybridation entre l'amidonnier et une herbe sauvage.

Dans les millénaires qui ont précédé notre ère, d'autres plantes domestiquées ailleurs sont arrivées peu à peu en Europe. C'est le cas des millets Panicum et Setaria, du chanvre, de l'oignon et du sésame en provenance d'Asie centrale, et du melon et de la pastèque en provenance d'Afrique.

C'est aussi le cas des arbres fruitiers, qui, biologiquement, sont fortement allogames*; la fixation de types à fruits agréables à consommer ne peut se faire que par multiplication végétative, à quelques exceptions près. Les arbres fruitiers mentionnés dans la Bible correspondent à la première vague de domestication, vers 4000 av. J.-C., soit bien après celle des plantes fondatrices. Ces espèces partagent la caractéristique de pouvoir être propagées par bouturage (caroubier, figuier, grenadier, mûrier noir, olivier, vigne, châtaignier) ou par graines (amandier, noyer). Un peu avant notre ère, l'invention du greffage a permis à d'autres espèces d'arriver par l'Asie centrale: pommier, poirier, prunier, cerisier, puis pêcher, abricotier, cognassier.

Les légumes ont été cultivés tardivement, du fait de leurs importants besoins en eau, qui nécessitent l'usage de l'irrigation. Ce n'est guère qu'à l'époque gréco-romaine qu'on les voit apparaître à l'état vraiment 
cultivé. Un certain nombre d'entre eux est originaire du bassin méditerranéen : chou, laitue, chicorées, radis.

\section{De l'Antiquité au Moyen Âge}

Dans l'Antiquité, les plantes n'ont cessé d'arriver dans le bassin méditerranéen, depuis l'Asie (cédrat, concombre) ou l'Afrique («haricot» de l'Ancien Monde [Vigna], gourde Lagenaria dont les jeunes fruits se mangent comme des courgettes). En colonisant une bonne partie de l'Europe, les Romains ont fortement contribué à l'élargissement de la gamme alimentaire, que ce soit par le commerce ou la culture. À partir du VII siècle, l'expansion de l'Islam a mis en communication l'Orient et l'Occident, de l'Andalousie au Proche-Orient, mais aussi de la Perse à l'Inde et à la Malaisie. Voyageurs, savants, commerçants ont pu diffuser les plantes et les savoir-faire agricoles qui leur étaient liés. Au fil des siècles, on a ainsi vu arriver dans la Méditerranée un grand nombre d'agrumes (citron, orange amère, pamplemousse vrai), le riz, le chou-fleur, l'épinard, l'aubergine.

L'Italie a joué un grand rôle dans les progrès de l'horticulture, et de nombreux fruits et légumes y ont été créés ou améliorés, comme le choufleur, le brocoli, le fenouil, les laitues et chicorées, des agrumes. L'Europe du nord a par contre joué un rôle très faible dans l'introduction des plantes. On ne lui doit guère que l'angélique, le raifort et la ciboulette. Vers le XII ${ }^{e}$-XIII ${ }^{e}$ siècles, le colza et le rutabaga semblent avoir été sélectionnés l'un en Flandre, l'autre en Suède. En revanche, avec l'essor de l'horticulture en Allemagne, aux Pays-Bas et au Danemark, de nouvelles variétés de légumes (choux, laitues) ont été sélectionnées et se sont répandues partout.

\section{Les grandes découvertes}

Après Christophe Colomb, l'expansion européenne dans le monde entier a entraîné un gigantesque brassage des plantes cultivées. Les plantes introduites en Europe ont été pour l'essentiel des plantes américaines. La raison tient à la biogéographie des découvertes. L’Amérique du Nord a un climat tempéré, et n'offrait donc pas d'obstacle particulier. Au Mexique et en Amérique du Sud, nombre d'espèces proviennent de zones d'altitude, et sont donc relativement tolérantes au froid. Les Espagnols ont été les passeurs de la majorité de ces plantes: maïs, patate douce puis pomme de terre, courges, tomate, piment et poivron, haricots Phaseolus, tournesol, arachide. Que les plantes d'origine américaine soient annuelles ou non, il a cependant fallu plusieurs siècles pour qu'apparaissent des variétés adaptées à nos jours longs, ce qui explique largement le temps mis par la pomme de terre ou la tomate pour remonter en latitude.

Quelques autres peuples ont également contribué aux introductions de plantes, comme les Français avec l'ananas (de Cayenne) et le topinambour (du Canada). Quant aux Portugais, ils ont surtout colonisé des régions tropicales chaudes, qui n'avaient guère de plantes adaptables en Europe. Ce n'est qu'après avoir atteint la Chine et le Japon qu'ils ont eu accès à l'orange douce ou à la nèfle du Japon.

\section{L'époque coloniale}

Soucieux d'exploiter au mieux les ressources des pays conquis, les Européens ont systématisé l'inventaire des ressources végétales, ce qui a permis l'essor d'une science comme la botanique et l'implantation de nombreux jardins botaniques, précurseurs de nos centres de recherche. Mais l'intention n'était pas seulement d'introduire des plantes cultivables en Europe. Il s'agissait surtout de développer des productions dans les colonies. On a ainsi vu se mettre en place un système de plantations, dont la canne à sucre est le symbole. Les oléagineux (arachide, coprah, palmier à huile) ont aussi pris une grande importance, au point qu'un pays comme la France a négligé la production de ce qu'on appelle encore aujourd'hui les «oléagineux métropolitains ». Ceux-ci (colza, tournesol) n'ont été promus qu'après l'indépendance des pays colonisés dans les années 1960 .

L'époque coloniale a également été marquée par un enthousiasme des élites pour l'introduction des plantes. Une société d'acclimatation était très active en France, mais à l'exception du crosne et de l'igname de Chine, rares ont été les succès, car ces nouvelles plantes étaient rarement adaptées aux conditions françaises, et on ignorait tout de l'amélioration des plantes.

\section{L'évolution intra- spécifique}

L'évolution génétique des espèces cultivées a été très profonde et rapide, et de nombreux types nouveaux de variétés sont apparus au sein d'espèces anciennes. On date par exemple l'obtention de choux 
pommés (blancs, rouges ou frisés) et de betterave à grosse racine d'après l'an Mil, celle du chou de Bruxelles du XVIII ${ }^{e}$ siècle et celle de l'endive du XIX ${ }^{e}$. Quant aux melons, leur évolution a été tellement profonde que nous aurions peine à reconnaître et apprécier les types anciens, qui étaient bien moins sucrés, et parfois utilisés immatures comme des concombres. De même, les poires fondantes d'aujourd'hui n'apparaissent guère qu'au XVIII ${ }^{\mathrm{e}}$ siècle.

Depuis le XIX ${ }^{\mathrm{e}}$ siècle, des sélectionneurs ont entrepris de rassembler des collections de variétés, puis de les croiser entre elles de façon raisonnée. L'essor de la génétique au $\mathrm{XX}^{\mathrm{e}}$ siècle a permis de créer de nouvelles espèces et variétés répondant aux besoins du marché et à l'évolution des systèmes de production. Depuis les années 1960, on voit ainsi apparaître des légumes de toutes les couleurs, produits de la sélection moderne.

La première plante obtenue pour des besoins industriels est en fait la betterave à sucre, qui a été sélectionnée à partir de la betterave fourragère dès le début du XIX ${ }^{\mathrm{e}}$ siècle. Le Blocus continental devait l'installer comme un substitut du sucre de canne. Une autre plante industrielle est le soja, qui n'a pu être cultivé en dehors de l'est de l'Asie qu'après la découverte de son association avec une bactérie fixatrice d'azote, au début du $\mathrm{XX}^{\mathrm{e}}$ siècle.

\section{La mondialisation}

Si la mondialisation des plantes alimentaires est plurimillénaire, elle s'est accélérée depuis le $\mathrm{XX}^{\mathrm{e}}$ siècle. La contribution des États-Unis s'est accrue, qu'il s'agisse de plantes exotiques cultivées (comme le pamplemousse moderne, cultivé en Floride, l'ananas ou la banane) ou de plantes domestiquées sur place (noix pacane, myrtilles américaines, mûres hybrides) ou améliorées (prunes japonaises, nectarines). De nos jours, de nombreux autres pays ont mis au point de nouvelles cultures, et le commerce international s'est considérablement développé. On connaît le kiwi de Nouvelle-Zélande et l'avocat d'Israël, mais avec les flux migratoires, de nouveaux marchés se sont créés et l'on voit déferler de nombreux produits asiatiques, africains ou américains.

De nos jours, la distinction entre produits disponibles par le commerce ou par culture locale tend à perdre son sens, car les productions se déplacent vers les lieux où elles sont produites à moindre coût. La moutarde de Dijon par exemple, produit emblématique de la France, est essentiellement fabriquée avec des graines de moutarde du Canada, et la plupart de nos légumes de conserve sont produits en Chine ou en Inde.

Les consommateurs, qui voyagent de plus en plus, sont attirés par des produits nouveaux, qui arrivent nimbés de qualités nutritionnelles (acérola, canneberge, goji) ou d'une origine culturelle prestigieuse (quinoa, amarante). Ils sont aussi attirés par les « légumes oubliés » et les variétés anciennes.

\section{Références bibliographiques}

- Harlan J.R., Les plantes cultivées et l'homme, Éditions CILF/ACT, Paris, 1987.

- Haudricourt A.-G., Hédin L., L'Homme et les plantes cultivées, Métailié, Paris, 1987.

- Maurizio A., Histoire de l'alimentation végétale depuis la Préhistoire jusqu'à nos jours, Payot, Paris, 1932.

- Zohary D. et al., Domestication of Plants in the Old World. The origin and spread of domesticated plants in Southwest Asia, Europe, and the Mediterranean Basin, $4^{\mathrm{e}}$ éd., Oxford University Press, 2012; trad. fr. à paraître chez Actes Sud, Arles. 


\title{
2.2. L'impact des évolutions de l'agriculture sur la qualité des matières premières
}

\author{
Joël Abecassis et Xavier Reboud
}

Avec l'exode rural qui a suivi la Seconde Guerre mondiale, les filières agricoles et alimentaires se sont d'abord organisées pour écouler les produits agricoles et assurer la nourriture des villes. Après la satisfaction des besoins quantitatifs, elles ont cherché à améliorer la qualité des produits destinés à notre alimentation. Au niveau agricole, des outils de tri ont été développés pour identifier parmi les ressources génétiques, les plus aptes à entrer dans les chaines de transformation. Parallèlement, les itinéraires agronomiques permettant de mener aux caractéristiques recherchées se sont généralisés.

Toute cette démarche a conduit à une amélioration certaine de la qualité des matières premières agricoles et à une réduction de leur variabilité qualitative. Cette standardisation normative a notamment pu reposer sur un système de bonifications/ réfactions appliqué lors de la collecte des matières premières agricoles. Elle s'est aussi traduite au niveau agronomique par une «artificialisation du milieu ». Les parcelles et les intrants ont été toujours mieux ajustés de façon à obtenir les propriétés fonctionnelles recherchées en ciblant la couverture des besoins physiolo- giques des plantes. L'amélioration génétique a soutenu une certaine standardisation qui s'est traduite par une capacité accrue à valoriser l'apport de nutriments au prix d'une certaine réduction de la diversité.

Toutefois, ce modèle agricole intensif basé sur la recherche d'un rendement le plus élevé possible tout en respectant les attentes de la qualité devient très dépendant de la fourniture d'intrants parfois mal valorisés par les cultures et sources de contamination des nappes phréatiques. Ceci n'est pas sans conséquence sur l'environnement. Aussi, sous la pression des acteurs de l'environnement, la demande de «verdir» notre agriculture s'impose progressivement même s'il reste des dissensions sur l'ampleur des externalités générées par l'agriculture et sur l'horizon auquel cet objectif peut être atteint.

\section{L’agroécologie}

La récente mise en place de la démarche d'agroécologie, prise ici au sens d'un ensemble de conduites qui vise à limiter les intrants en tirant au mieux profit de processus biolo- giques existants, pose des questions clefs pour les filières de production agricole. D'abord sur les incidences en termes de volumes disponibles en relation avec les critères économiques de faisabilité et de compétitivité des productions, ensuite autour de la variabilité croissante de la qualité des matières premières issues de l'agriculture.

Les premières démarches relevant de l'agroécologie en milieu tempéré et des similitudes avec d'autres modes de conduite en agriculture biologique ou en protection intégrée* permettent de faire quelques projections. Les volumes des matières premières pourraient baisser légèrement avec une moindre capacité à atteindre les rendements les plus forts dans des conditions plus souvent sub-optimales de couverture des besoins des cultures. Ceci restera de moindre ampleur que la variation interannuelle desdits volumes. Également exploitée en agroécologie, la diversification de l'assolement* (Figure 1), destinée à rendre le milieu moins prévisible et moins invasif aux ravageurs et pathogènes, fait aussi une plus large place aux cultures de légumineuses (féverole, luzerne) ainsi qu'aux cultures à visée énergétique dans la 
mosaïque paysagère (Miscanthus, ou taillis de robinier). Les choix variétaux se portent parallèlement sur des variétés très productives mais présentant de bonnes caractéristiques de résilience* face aux fluctuations d'un milieu moins artificialisé et face à une protection phytosanitaire* plus parcimonieuse.

En matière de qualité, il est peu vraisemblable qu'une démarche d'agroécologie affecte en profondeur la qualité nutritionnelle des produits; c'est d'ailleurs ce que suggèrent les analyses comparatives de composition entre les modes de production. À l'exception des effets connus d'incidence de la fertilisation, les diffé- rences semblent relever d'abord des caractéristiques variétales plus que des modalités de conduite.

Néanmoins, la réduction des intrants et en particulier de l'azote, pourrait impacter les propriétés sensorielles des aliments via la modification de leur composition. Cela concerne principalement les produits céréaliers (qualité culinaire des pâtes alimentaires) et dans une moindre mesure certaines productions fruitières (teneur en polyphénols). On s'interroge davantage sur les conséquences possibles en matière de qualité sanitaire. S'il est admis que la réduction d'emploi des pesticides devrait s'accompa- gner d'une réduction sensible de la teneur en ces contaminants dans nos aliments, l'impact de produits de substitution pouvant être utilisés à forte dose (par exemple sur la teneur en cuivre) est encore un objet de recherche. Par ailleurs, on sait mal prédire les conséquences de la levée d'un système répressif sur la recrudescence de certains pathogènes (ex: ergot de seigle*, carie du blé*), sur le développement précoce au verger de maladies qui affecteront les possibilités de conservation avant commercialisation ou transformation ou encore sur la prévention de nouveaux contaminants biotiques* favorisés par le réchauffement climatique.

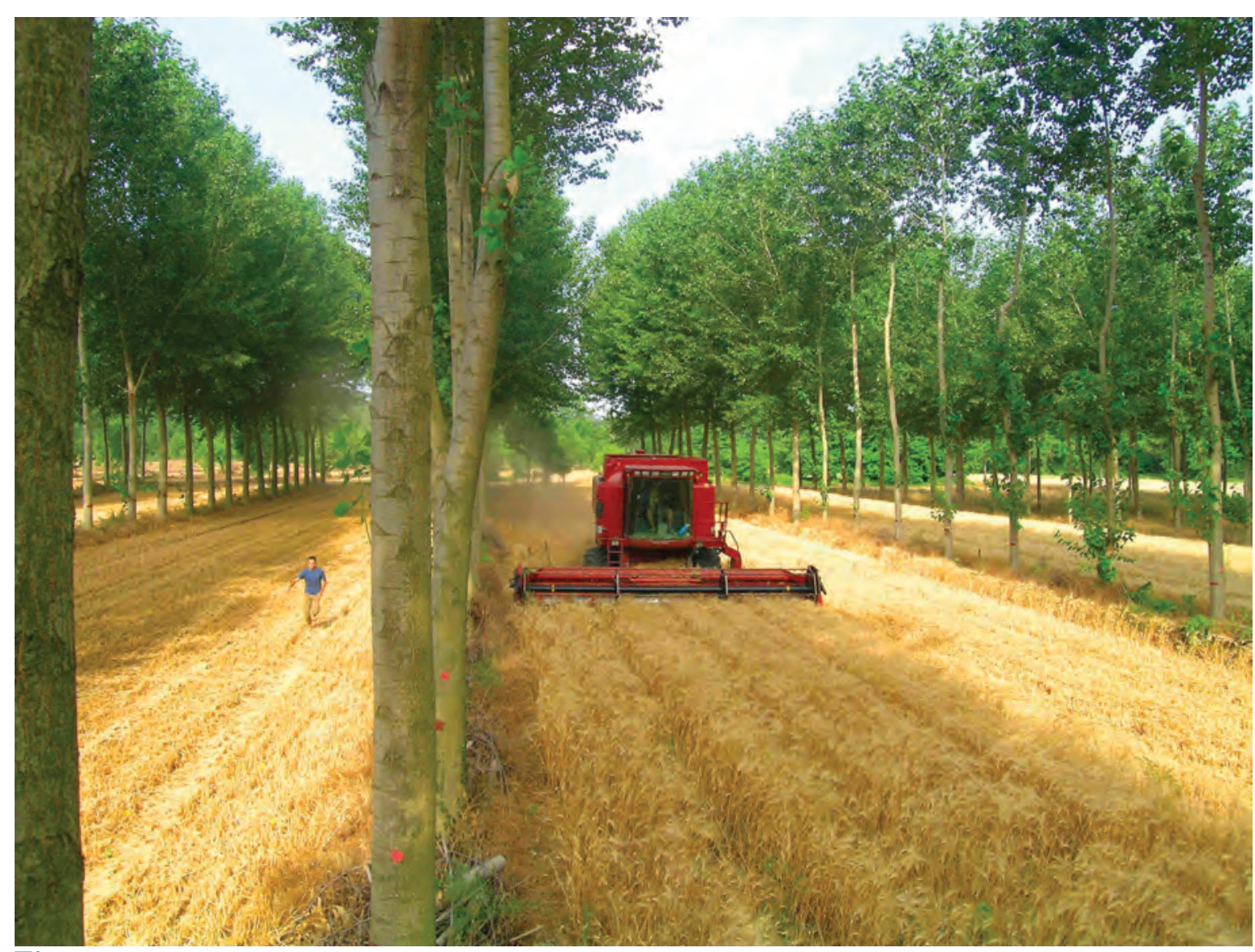

Fig. 1 - Moisson de blé dur dans une parcelle agroforestière expérimentale adulte près de Vézenobres. La complémentarité des arbres et des cultures permet aux parcelles d'être plus productives. L'arbre et la bande de sol non cultivée à son pied abritent de nombreuses espèces animales et végétales qui peuvent contribuer à la protection des cultures contre les ravageurs (photo Christian Dupraz/INRA). 


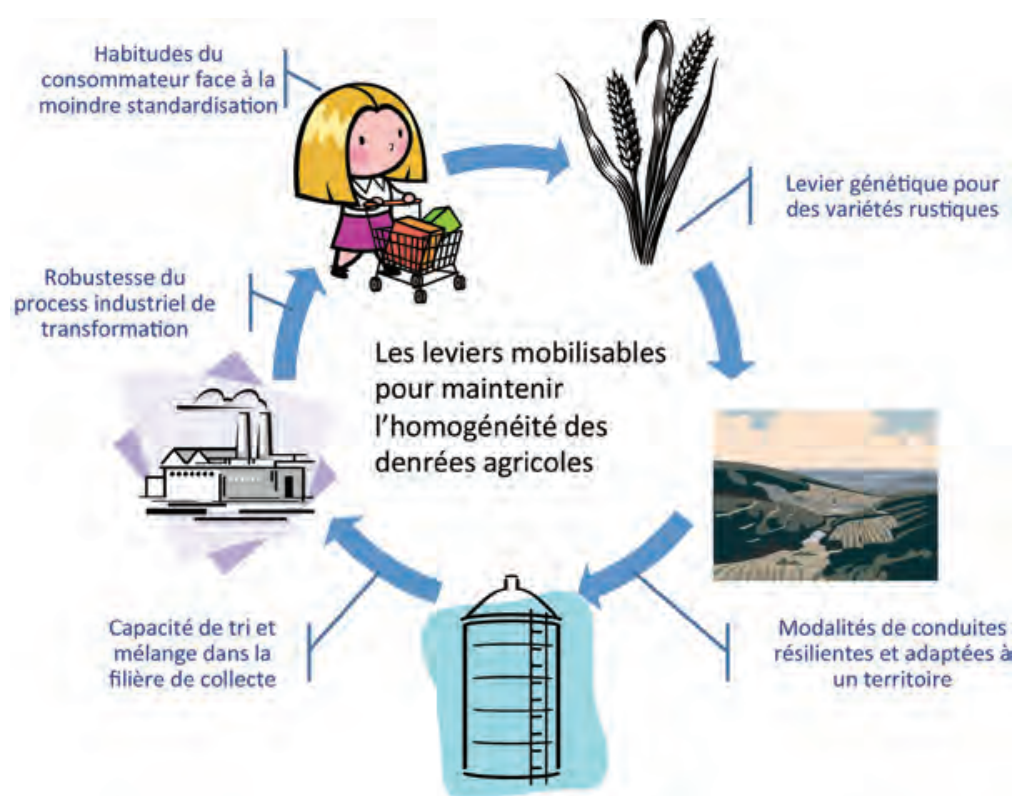

Fig. 2 - Du champ à l'assiette: un synoptique des leviers mobilisables pour maintenir la production et la qualité des matières premières agricoles.

L'effet principal de ce changement de pratiques va se traduire par un fort accroissement de l'hétérogénéité et de la variabilité de la composition et des propriétés d'usage des matières premières. Des travaux sont déjà engagés pour chercher à réduire cette variabilité en développant de nouveaux outils et méthodes de pilotage précis des cultures.

Les filières devront néanmoins revoir leur stratégie au niveau de la collecte et du stockage. Va-t-on chercher à constituer par mélange des lots aussi homogènes que possible mais avec une dépense énergétique accrue ou aller plutôt vers une meilleure segmentation des marchés avec des prix différenciant davantage les qualités des matières premières pour mieux rémunérer les démarches de qualité des producteurs ? Ces stratégies devront se définir dans le cadre des contraintes des marchés internationaux en cherchant à maintenir la compétitivité des productions finis dont les propriétés organoleptiques répondent aux attentes des consommateurs. En réduisant l'apport d'azote sans compensation par des blés à plus forte teneur en protéines, le risque peut donc être accru d'une perte de compétitivité de nos productions sur les marchés internationaux.

Au niveau national, les conséquences pour les filières pourraient être d'ampleur plus limitée si l'ensemble de la chaîne est mise à contribution pour contenir l'accroissement prévisible de la fluctuation des volumes et de la composition (Figure 2). Cela crée de nouveaux besoins de recherche nécessitant la mobilisation simultanée de plusieurs leviers: variétés présentant des caractéristiques pour leur capacité à corriger les déficits à la norme de transformation, pratiques agronomiques plus parcimonieuses et précises exploitant de nouveaux capteurs et outils de pilotage embarqués, usage systématique d'analyse en ligne pour favoriser le tri dès le stockage et la formulation de lots aux propriétés en adéquation avec les capacités d'adaptation de la chaîne de transformation. Le consommateur sera sans doute aussi amené à s' habituer à une moindre standardisation de ce qu'il consomme.

\section{Références bibliographiques}

- Cassman K.G., «Ecological intensification of cereal production systems: Yield potential, soil quality, and precision agriculture», Proc Nat Acad Sci USA 1998; 96: 5952-59.

- Francis C. et al., "Agroecology: The ecology of food systems", J Sust Agric 2003; 22: 99-118.

- Guyomard H., Vers des agricultures à hautes performances. Vol 1: Analyse des performances de l'agriculture biologique, Inra, Paris, 2013.

- Seufert V. et al. «Comparing the yields of organic and conventional agriculture», Nature 2012; 485: 229-32. 


\subsection{Les ressources marines: un enjeu pour l'alimentation}

Monique Étienne et Jean-Pascal Bergé

Exploiter, produire et utiliser les ressources halieutiques pour répondre aux besoins alimentaires humains, qualitatifs et quantitatifs, tout en respectant les règles d'un développement durable, implique de nombreux facteurs et nécessite le concours de tous les acteurs sociaux et économiques ainsi qu'un investissement dans la recherche.

\section{Produire la quantité nécessaire pour}

\section{répondre aux besoins nutritionnels}

La population mondiale ne cesse d'augmenter (7,2 milliards en 2013) et la consommation de poisson par habitant a doublé en 50 ans: elle atteint aujourd' hui $18,6 \mathrm{~kg}$ (équivalent poids vif).

Le premier enjeu est pour la pêche de conserver un niveau constant et la diversité des espèces. La production mondiale des pêches est stable depuis 20 ans à environ 90 millions tonnes/an $(\sim 80 \mathrm{Mt}$ marine, en diminution, et $\sim 10 \mathrm{Mt}$ continentale, en augmentation). La majorité des stocks des 10 principales espèces pêchées ( 30\% des captures marines mondiales) est pleinement exploitée sans possibilité d'accroissement de production, certains stocks étant même menacés (surcapacité des flottilles thonières). Toutefois, la mise en œuvre de plans efficaces devrait permettre d'augmenter ou de restaurer la production de stocks surexploités. C'est le cas par exemple de l'immersion de récifs artificiels* ${ }^{*}$ utilisés au Japon mais à un stade préliminaire en France. Cette simulation de zone rocheuse favorable à l'accroissement et à la protection de la ressource halieutique constitue une réponse aux pressions exercées par les activités humaines, dégradations environnementales et surpêche, en particulier dans les zones côtières. Les deux défis majeurs sont de lutter contre la pêche illicite par la traçabilité des produits de la pêche du « filet à l'assiette » et d'accéder à la ressource malgré l'augmentation du coût de l'énergie.

Pour l'aquaculture, l'enjeu est d'accroître la production tout en respectant l'environnement. En 50 ans la production aquacole mondiale est passée d'un niveau négligeable à 70 millions de tonnes/ an; elle est majoritairement d'eau douce $(\sim 70 \%)$ et essentiellement asiatique (89\%). L'aquaculture est consommatrice de farine et d'huile de poisson principalement issues de pêche minotière*, néanmoins en baisse régulière avec l'introduction de protéines végétales notamment.

La mariculture (aquaculture en eau de mer) est dépendante des conditions environnementales et demeure sensible aux maladies, qui ont causé récemment des pertes importantes.

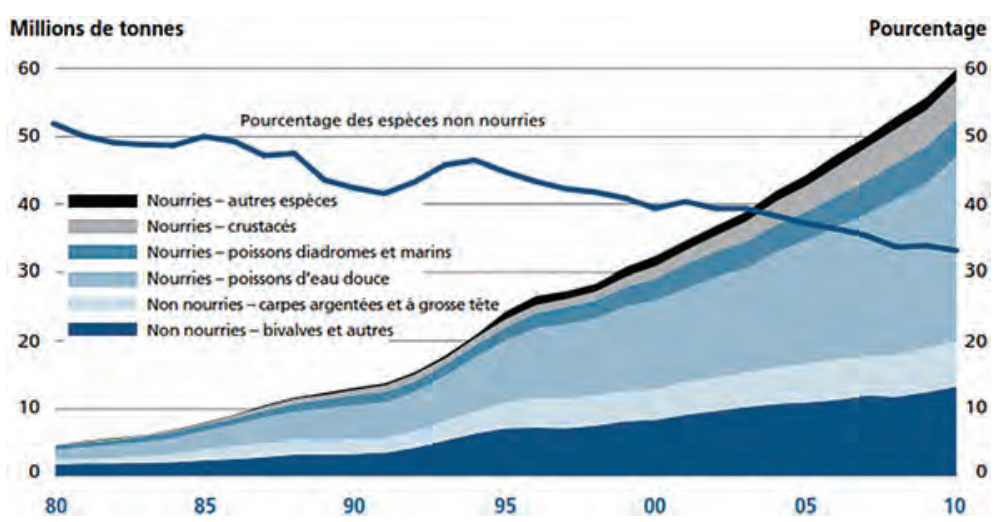

Fig. 1 - Production aquacole mondiale d'espèces non nourries et d'espèces nourries. 
L'implantation des élevages s'est surtout faite dans des zones abritées et peu peuplées, mais dans un contexte d'expansion il convient d'éviter des conflits d'usage tout en minimisant l'impact environnemental. Deux solutions sont envisagées: des installations à terre en circuit fermé (avec recyclage des eaux) et des élevages offshore de type aquaculture multitrophique intégrée (AMTI) qui associent l'élevage de plusieurs espèces complémentaires (algues, mollusques, crustacés et poissons) et maximisent la productivité.

Au regard de ces contraintes, positionner les élevages à une distance raisonnable des lieux de consommation et mettre sur le marché des produits sains constituent une gageure.

\section{Utiliser les ressources}

\section{afin de satisfaire}

\section{la demande à des}

\section{conditions et prix acceptables}

Les évolutions des préoccupations nutritionnelles, des comportements humains et du prix du poisson doivent conduire à modifier les pratiques.

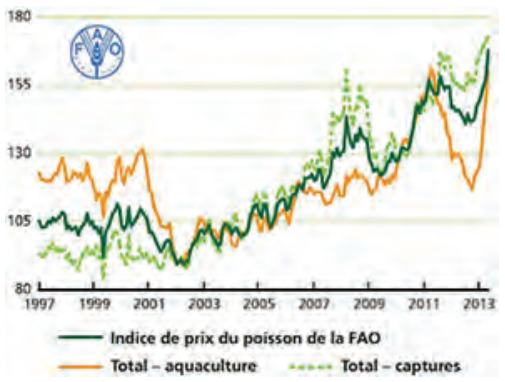

Fig. 2 - Indice des prix du poisson de la FA0 (2002-2004=100) (Source: Norwegian Seafood Council)
Il faut d'abord réduire pertes et gaspillages. De nombreuses espèces halieutiques pêchées ne sont pas utilisées (captures accessoires, retraits, invendus) car méconnues ou peu prisées; (ré)-apprendre aux consommateurs la diversité des espèces, leurs variations saisonnières et leurs modes de préparations, voire développer des circuits courts adaptés devrait contribuer à réduire ces déficits. D'autre part, la filière alimentaire marine est sujette à de nombreuses pertes dont les raisons differrent selon les territoires: dans les pays en développement elles sont surtout liées au stockage, aux difficultés de transport, à l'infestation par des insectes et dans les pays développés les rejets sont d'ordre normatif, qualitatif et sanitaire. L'utilisation des techniques existantes et innovantes (biopréservation*, atmosphère modifiée...) et la limitation des trajets entre les lieux de production et de consommation contribueront à réduire le développement des micro-organismes pathogènes et altérants, donc à réduire les rebuts. Parallèlement, la maximisation de la fraction consommable (la chair ne représente que 40 à $70 \%$ du poids vif) par une autre mise en valeur des sous-produits que l'alimentation animale (en France $96 \%$ des sous-produits sont transformés en farine et hachis) pourrait à terme réduire le coût des produits alimentaires.

Il s'agit aussi de produire propre. Le traitement des effluents et la prise en compte du bilan carbone sont devenus incontournables pour les industries de transformation dont la pérennité est également liée au commerce international et aux régimes douaniers (le thon en particulier).

Il faut également innover pour proposer des produits répondant aux besoins des consommateurs. Les produits de la mer bénéficient d'une bonne image (fraîcheur, santé) mais présentent de nombreux freins à l'achat (temps de préparation, arêtes, prix, manque de praticité); pour y remédier il faudra continuer à innover : poisson cru fermenté type saucisson sec, emballage intelligent, etc. En réponse à une demande croissante, le choix de nouvelles espèces aquacoles devra dépendre de leurs potentialités d'utilisation: durée de conservation, rendement de chair, consommation en cru ou cuit, modes de traitement, acceptation par le consommateur, qualité sensorielle. Par exemple l'élevage des mollusques bivalves pourrait être diversifié s'il était associé à une commercialisation sous forme décoquillée prête à préparer ou à consommer.

Enfin, il convient d'utiliser et de valoriser les ressources végétales marines, en développant des ressources telles les algues pour des finalités alimentaires comme cela se pratique en Asie, mais aussi en mettant en avant leurs propriétés nutritionnelles (richesse en acides aminés essentiels, forte teneur en protéines).

\section{Références bibliographiques}

- FA0, La situation mondiale des pêches et de l'aquaculture, SOFIA, 2012.

- Les cahiers de FranceAgriMer /chiffres-clés / Pêche et aquaculture en France, 2013.

- http://www.spc.int/fame/doc/meetings/June2012_Fisheries

Meetings/Dans_le_poisson_tout_est_bon.pdf

- http://www.bibliomer.com/documents/fiches/Coproduits_vf.pdf 


\subsection{L'itinéraire technologique pour fabriquer un aliment: un choix à critères multiples}

\section{Paul Colonna et Jean Tayeb}

Chaque aliment est issu d'une ou plusieurs matières premières agricoles, auxquelles des opérations de transformation sont appliquées. L'ensemble des opérations de production des matières premières et de transformation contribue à l'élaboration des caractéristiques de l'aliment. L'apport de la transformation est nécessaire dans la mesure où les matières premières agricoles se conservent pendant un temps limité, possèdent des caractéristiques variables et sont de qualité nutritionnelle irrégulière; les technologies agro-alimentaires les transforment en denrées alimentaires sûres, plus stables, de bonne valeur sensorielle et en général nutritionnelle.

\section{Les variables d'analyse}

La qualité de chaque aliment peut être décrite par huit variables, sensorielle (appelée aussi organoleptique), nutritionnelle, sécurité, coût, praticité, image, environnementale et éthique. Pour chaque objectif choisi de caractéristiques finales (c'est-à-dire le positionnement visà-vis de ces huit variables), il existe un choix raisonné d'opérations et de valeurs paramétriques. Les opéra- tions à caractère additif (un emballage, par exemple) ou soustractif (un tri, par exemple) peuvent être décrites assez facilement sur l'ensemble de l'itinéraire technologique. D'autres en revanche créent des propriétés nouvelles car les éléments de l'aliment sont en interactions: la compréhension des propriétés de l'ensemble dépasse celle des propriétés des parties constitutives. L'absence d'interaction, par exemple le fait de conserver un contraste de texture dans le même aliment, représente aussi une propriété nouvelle, qui s'oppose au principe des transferts de masse par diffusion dans un aliment. Apparaissent ainsi les notions de niveau d'organisation (Figure 1) et de propriété émergente, à la base de l'analyse de la complexité des aliments, comme le montre l'exemple de l'itinéraire technologique du pain français qui illustre comment l'existence d'une organisation multi-échelle renvoie à des mécanismes différents d'établissement de chaque niveau, avec des cinétiques différentes (Tableau). En raison de la complexité des aliments, il est en effet impératif de développer des approches multi-échelles qui identifient et représentent à la fois les agents essentiels de la réaction et l'environnement réactionnel nécessaire. Ces approches multi-échelles sont aussi indispensables pour relier les observations macroscopiques à des propriétés microscopiques de la matière.

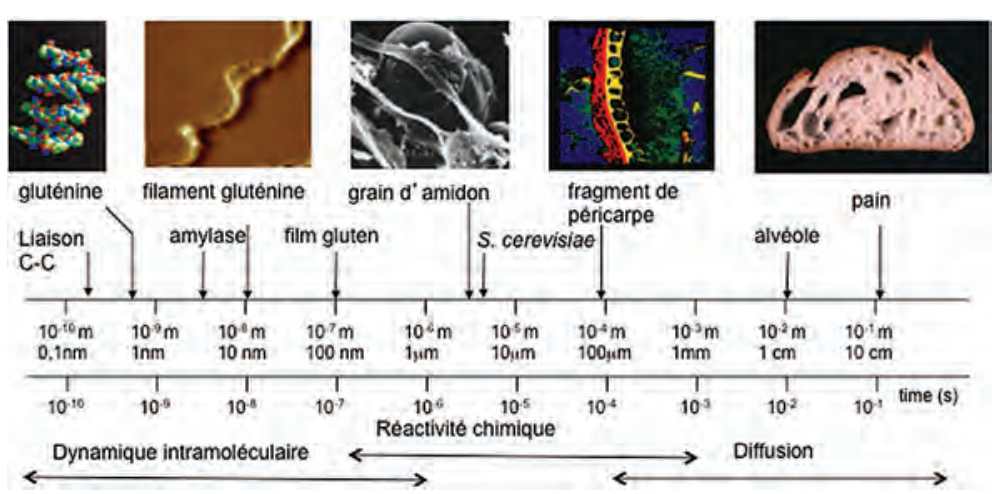

Fig. 1 - Les échelles de structure et de temps caractéristiques dans un aliment céréalier. 
Tableau 1 - Itinéraire technologique du pain français

\begin{tabular}{|c|c|c|}
\hline Étape & Phénomènes constitutifs & $\begin{array}{l}\text { Principale propriété } \\
\text { concernée pour le pain final }\end{array}$ \\
\hline Choix de la variété de blé & Aptitude du gluten (gliadines et glutenines)* à la panification & $\begin{array}{l}\text { Propriété sensorielle (aspect } \\
\text { et texture finale du pain) }\end{array}$ \\
\hline Production agricole & $\begin{array}{l}\text { Accumulation des molécules constitutives, en particulier } \\
\text { amidon, gluten, microconstituants } \\
\text { Maîtrise de la formation des mycotoxines et de la présence } \\
\text { résiduelle de produits phytosanitaires }\end{array}$ & $\begin{array}{l}\text { Propriété nutritionnelle } \\
\text { Sécurité sanitaire }\end{array}$ \\
\hline $\begin{array}{l}\text { Meunerie } \\
\text { (broyage et tri) }\end{array}$ & $\begin{array}{l}\text { Elimination des fractions tissulaires et chimiques les moins } \\
\text { intéressantes aux plans nutritionnel, sensoriel et technologique } \\
\text { (révélé lors du pétrissage) } \\
\text { Endommagement de l'amidon nécessaire pour sa capacité à } \\
\text { fermenter } \\
\text { Maîtrise de la présence résiduelle de produits phytosanitaires } \\
\text { Libération des contenus intracellulaires nécessaire pour la } \\
\text { réactivité supramoléculaire du gluten }\end{array}$ & $\begin{array}{l}\text { Propriété nutritionnelle } \\
\text { Sécurité sanitaire }\end{array}$ \\
\hline $\begin{array}{l}\text { Pétrissage } \\
\text { (travail mécanique } \\
\text { facilitant des réactions } \\
\text { physico-chimiques) }\end{array}$ & Création d'un réseau protéique (propriété de texture émergente) & Propriété sensorielle \\
\hline $\begin{array}{l}\text { Fermentation } \\
\text { (action des levures) }\end{array}$ & $\begin{array}{l}\text { Alvéolation du réseau (propriété de texture émergente) } \\
\text { Création de molécules aromatiques }\end{array}$ & Propriété sensorielle \\
\hline Cuisson & $\begin{array}{l}\text { Dénaturation des protéines, gélatinisation de l'amidon (propriété } \\
\text { émergente) et stabilisation du réseau } \\
\text { Formation de la mie et de la croûte, et donc d'un contraste de } \\
\text { texture } \\
\text { Création de molécules aromatiques (réaction de Maillard*) } \\
\text { (propriété émergente) }\end{array}$ & $\begin{array}{l}\text { Propriétés nutritionnelle } \\
\text { et sensorielle } \\
\text { Praticité }\end{array}$ \\
\hline
\end{tabular}

\section{La notion de filière}

Les liens entre les différents acteurs de l'itinéraire technologique définissent le concept de «filière » agricole et agro-alimentaire, qui permet de formaliser l'ensemble des opérations, flux de matière, d'énergie et d'informations et jeux d'acteurs intervenant à partir d'un produit agricole - et ce jusqu'au consommateur.

Pour chaque catégorie d'aliment, ces filières peuvent aussi s'analyser comme des chaînes de valeur en répertoriant la séquence des activités qui créent de la valeur, considérées du point de vue de chaque entreprise. Les chaînes de valeur ainsi constituées ont conduit à l'établissement de chartes de qualité (labels), garantissant des propriétés sensorielles et nutritionnelles minimales, le respect de considérations environnementales (agriculture biologique) ou éthiques (commerce équitable).

\section{L'évolution}

\section{des itinéraires}

\section{technologiques}

Les itinéraires technologiques sont par essence métastables: à chaque période, ils représentent un compromis entre l'état de l'art technique, les disponibilités des ingrédients, les attentes évolutives des consommateurs dans un segment de marché donné. Ils évoluent pour intégrer l'apparition de nouvelles technologies, de nouvelles attentes des consommateurs (Figure 2). Les impacts environnementaux et les considérations nutritionnelles sont aujourd'hui les forces motrices de ces changements.

En particulier, la transition énergétique conduit à développer une approche globale qui analyse l'utilisation de l'énergie et des ressources (eau). Au niveau industriel, l'intégration énergétique vise notamment à utiliser, voire à stocker, l'énergie thermique pour différents usages (notion de « cascade énergétique »). Elle repose sur des techniques d'analyse systémiques (analyses Pinch*, exergétique*, etc.) et est largement complétée par le concept 
d'intensification des procédés. Ce dernier désigne le développement de méthodes et de dispositifs innovants qui, en comparaison de l'existant, offrent une diminution de la consommation d'énergie, de la production de déchets et du rapport taille sur capacité tout en maintenant la qualité des produits. Ces approches font partie de l'éco-conception* des procédés.

La maîtrise des changements dans l'élaboration des produits alimentaires et de la conduite des procédés de transformation nécessite la construction d'outils adaptés d'aide à la décision. Différents concepts, compétences et outils méthodologiques innovants ont été développés pour faire « coopérer » et intégrer l'ensemble des informations ou connaissances ponctuelles capitalisées à différentes échelles et sous différents formats par les acteurs concernés. Deux champs de recherche complémentaires sont en particulier très actifs aujourd'hui: d'une part, l'acquisition de connaissances sur les interactions entre les constituants, notamment du point de vue de la dynamique (évolution

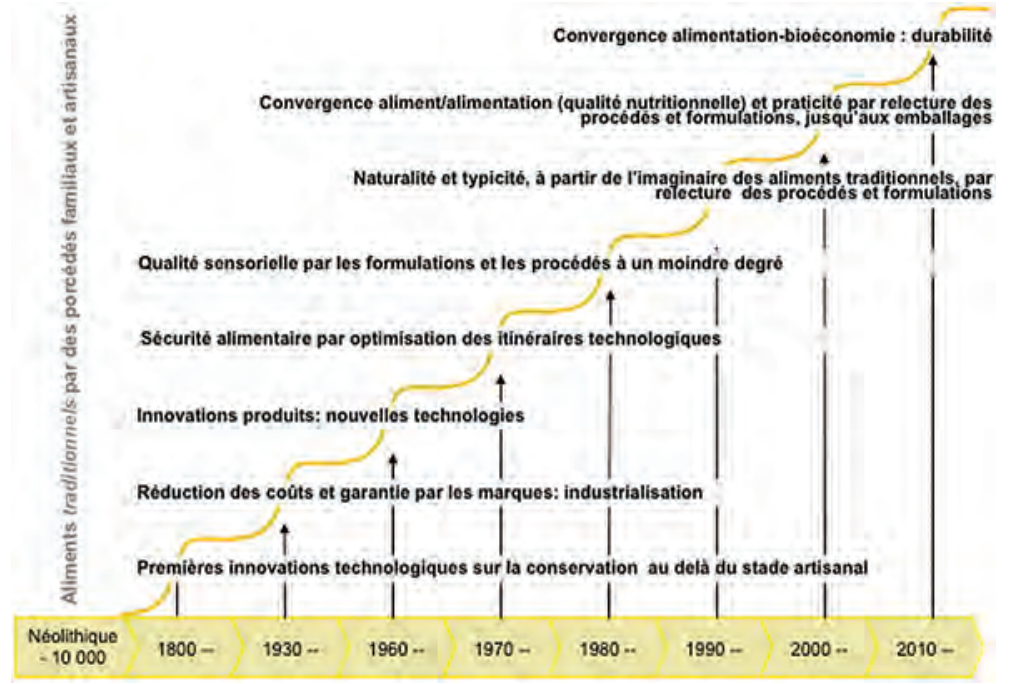

Fig. 2 - Grandes dynamiques historiques des itinéraires technologiques des aliments. au cours du temps) du système étudié; d'autre part, l'intégration des connaissances, y compris le savoir-faire des experts, pour déterminer l'ensemble des chemins qui permettent d'amener le produit à la cible (tube de viabilité; par exemple la «longue conservation » peut être obtenue de différentes façons: via l'emballage, via la cuisson prolongée, via l'acidification par des micro-organismes, etc.).

\section{Références bibliographiques}

- Belton P., The Chemical Physics of Food, Blackwell, Oxford, 2007.

- Bertolini A.C., Starches, characterization, properties and applications, CRC Press, Boca Raton, London-New York, 2011. 


\title{
2.5. Comment crée-t-on \\ une diversité de produits \\ à partir d'une matière unique? Le cas de la filière laitière
}

\author{
Joëlle Léonil et Gilles Garric
}

Le consommateur côtoie aujourd'hui plus de 3500 références de produits laitiers répartis dans six grandes familles : laits liquides, fromages, produits laitiers frais, crèmes, beurres et spécialités laitières, plus une pléthore d'ingrédients laitiers. Une telle diversité repose sur l'exceptionnelle plasticité de cette matière première, le lait, et les propriétés uniques de ses constituants. Elle est issue d'une maîtrise empirique plurimillénaire de la transformation du lait et de l'évolution récente des technologies.

\section{Le lait, un système}

\section{multiphasique \\ en équilibre}

Aliment complet et exclusif du nouveau-né mammifère, le lait possède des caractéristiques physiques et chimiques qui en font une matière première de premier ordre. Sur le plan chimique, c'est un fluide biologique qui renferme une multiplicité de molécules (protéines, lipides,

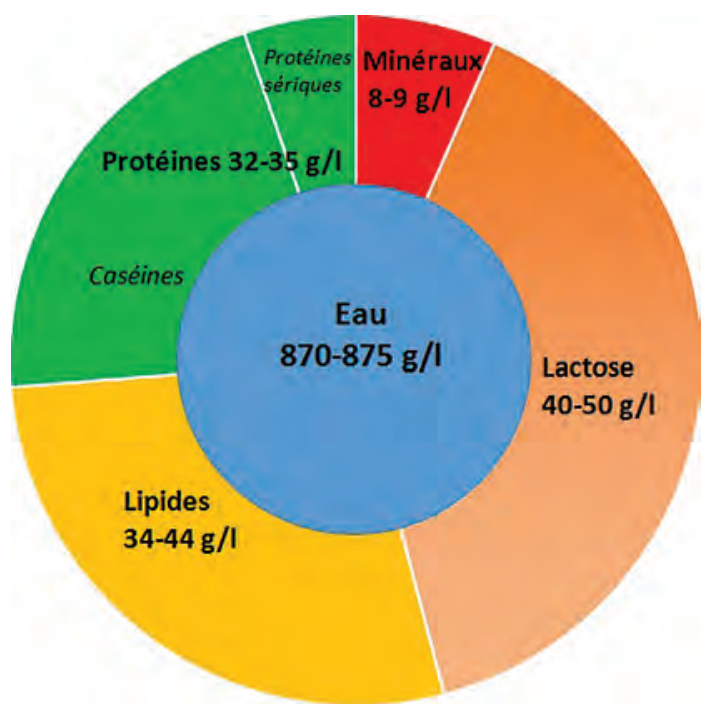

Fig. 1 - Composition du lait bovin. glucides, minéraux, vitamines) (Figure). Sur le plan physique, le lait est constitué de trois phases en équilibre: $1^{\circ}$ une phase aqueuse $(87 \%$ eau), le sérum du lait; $2^{\circ}$ une suspension particulaire formée d'assemblages de caséines (appelée micelle de caséines) (2.6\%), à l'origine du caillé fromager obtenu par coagulation suite à l'action de micro-organismes, d'enzyme ou d'acides; $3^{\circ}$ une émulsion (4.2\%) de type huile dans l'eau qui donne les corps gras laitiers.
L'origine de la diversité actuelle est la maîtrise technologique empirique de changements de phases du lait qui génère une très large palette de produits de textures différentes (liquide, gel). Ces produits sont dotés de propriétés nutritionnelles liées à la valeur intrinsèque de leurs constituants et d'un fort potentiel sensoriel puisque le lait est un excellent substrat pour le développement de flores utiles, aux capacités aromatiques très diversi- 
fiées. Cette histoire a déjà plusieurs millénaires.

Le deuxième volet a seulement 50 ans et repose sur la maitrise d'un certain nombre de procédés comme les traitements thermiques, le fractionnement du lait par les technologies à membranes (techniques de séparation physique basées sur la taille qui permet d'isoler les macromolécules constitutives du lait), ou le séchage par atomisation (méthode de déshydratation qui, appliquée à un liquide, le transforme en une poudre par passage dans un flux d'air chaud). Tout ceci ouvre l'accès à une palette de fractions laitières, qui autorisent une recomposition de novo pour créer de nombreux ingrédients ou matrices laitières.

\section{La dynamique d'apparition des grandes familles de produits laitiers}

Dès le début du Néolithique, la transformation du lait visait la conservation des éléments nutritifs afin d'en différer la consommation dans le temps et l'espace. En effet, le lait liquide (87 \% d'eau) est fragile, et ne peut se conserver à température ambiante. De manière très empirique, les premiers laits fermentés conservables quelques jours sont apparus par fermentation lactique et/ou alcoolique du lait: leben au
Moyen-Orient, kéfir dans le Caucase, koumis en Asie Centrale. À partir du III ${ }^{e}$ millénaire avant notre ère, la séparation de phase spontanée du lait, par simple décantation, a été exploitée pour récupérer les phases dites lourde (lait écrémé) et légère (crème) créant ainsi les premiers « laits fermentés égouttés ou fromages » tels que le labneh au Moyen-Orient ou le panir indien, puis les fromages pressés romains. La phase légère, barattée, éventuellement chauffée et clarifiée (comme le ghee indien) conduisit à la catégorie des produits gras telle qu'on la connait encore aujourd' hui : crèmes, beurres et matières grasses concentrées (huile de beurre et matière grasse laitière anhydre).

Des pratiques encore plus drastiques d'élimination de l'eau du lait pour concentrer les éléments nutritifs, améliorer la durée de consommation et faciliter le transport se sont ensuite développées : par des phases d'égouttage, de chauffage et d'affinage plus poussées, le lait a généré selon les terroirs des fromages aux formes, textures et goûts extrêmement diversifiés. Aujourd'hui les connaissances en microbiologie et sur les capacités aromatisantes des levains continuent d'être une source de diversification des produits finis de type fromage ou spécialités fromagères. C'est seulement

\section{Références bibliographiques}

- Jeantet R. et al., Science des aliments: Biochimie. Microbiologie. Procédés. Produits., Vol 1 et 2, Lavoisier, Paris, 2006. au XIX ${ }^{e}$ siècle que la conservation de lait sous forme liquide (laits de consommation) apparaît grâce à l'appertisation, la pasteurisation, puis dans les années 1970 , le traitement ultra haute température (UHT) qui est une technique de stérilisation où le lait est porté à haute température $\left(135^{\circ} \mathrm{C}, 1-2 \mathrm{~s}\right)$ puis immédiatement et très rapidement refroidi. Parallèlement le développement du séchage par atomisation boucle la boucle de cette éternelle recherche d'élimination de l'eau du lait ou de ses constituants afin d'en conserver le meilleur et de le transporter aisément.

Aujourd'hui la diversification se joue à l'échelle moléculaire et dans la recombinaison. Le fractionnement du lait par techniques à membrane, associé éventuellement à des procédés d'hydrolyse enzymatique a conduit à rechercher des fractions protéiques à haute valeur ajoutée (isolats et concentrés de protéines solubles, micelles de caséines, protéines ou peptides purifiés bioactifs) et dotées de propriétés biologiques. Cette évolution a été décisive dans la conception d'ingrédients fonctionnels qui ne cesse aujourd'hui de se raffiner pour créer des produits de plus en plus adaptés aux exigences multiples du consommateur (sécurité, goût, nutrition, santé, service et sens de l'acte d'achat), le tout dans un prix contenu. 


\title{
2.6. Le rôle des ingrédients et des produits alimentaires intermédiaires pour conférer des qualités aux aliments
}

\author{
Marc Anton
}

L'aliment transformé est un matériau complexe qui ne résulte pas de la simple juxtaposition de ses différents constituants, extraits de matières premières animales et/ou végétales, mais de leurs interactions et assemblages complexes sous l'impact des procédés thermo-mécaniques. Ces interactions permettent de construire des structures originales et d'exprimer ainsi des propriétés distinctives sur les plans sensoriel (texture, arômes, saveur) et nutritionnel. Ces aliments

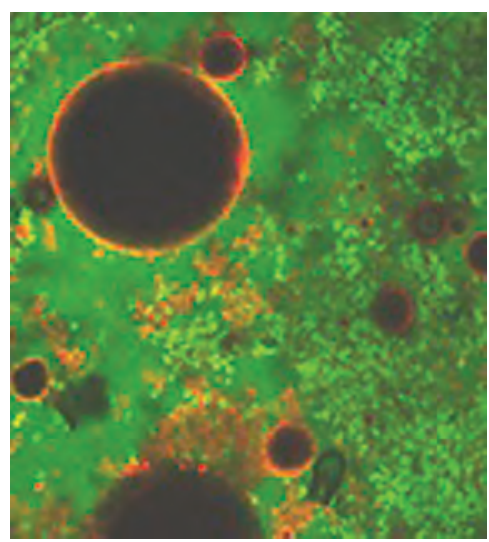

Fig. 1 - Ultrastructure d'une émulsion foisonnée (type crème chantilly); noir : bulles d'airs, rouge: gouttelettes de lipides, vert clair: protéines, vert foncé: polysaccharides. liquides ou solides, qui peuvent être compartimentés (mousses solides ou liquides, gels, émulsions huile-eau), sont souvent constitués de plusieurs phases présentant des caractéristiques physico-chimiques différentes (densité, potentiel chimique) ce qui génère une forte instabilité thermodynamique (Figure 1). De plus les caractéristiques de ces diverses phases peuvent être modifiées par des stimuli extérieurs (température, cisaillements*, pH, sels) et apporter ainsi au système une versatilité permettant de moduler les propriétés de l'aliment.

\section{Les ingrédients}

Dans ces systèmes complexes, évoluant avec le temps ou sous l'action de contraintes externes, le rôle des ingrédients est, d'une part, de garantir la structuration de ces aliments créée par les procédés thermo-mécaniques et, d'autre part, d'assurer leur stabilité physique et/ou chimique en fonction des conditions extérieures et sur la durée séparant leur fabrication de leur consommation. Ainsi, chaque famille d'ingrédients apporte une fonctionnalité particulière (émulsifiant*, foisonnant*, acidifiant, rehausseur de goût, antioxydant, conservateur, épaississant, gélifiant, colorant) mais bien souvent les ingrédients sont des agents multifonctionnels.

Sur un plan réglementaire, les ingrédients (protéines, polysaccharides*, lipides, etc.) doivent être énumérés sur l'étiquetage du produit dans l'ordre décroissant de leur importance pondérale et désignés par leur nom spécifique. Les ingrédients qui appartiennent à plusieurs catégories sont désignés par rapport à leur fonction principale. Les addi-

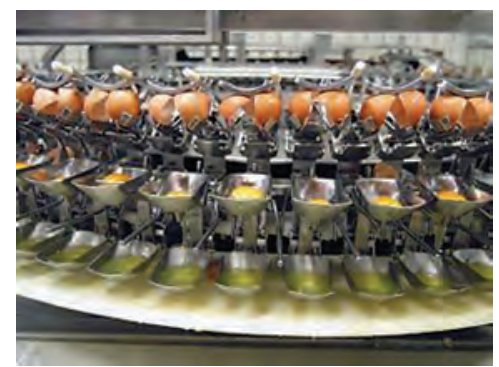

Fig. 2 - Cassage automatique et séparation mécanique du jaune et du blanc d'œuf. 
tifs et auxiliaires technologiques ne sont pas considérés comme ingrédients : les additifs sont des substances ajoutées aux ingrédients pour aider à la fabrication, alors que les auxiliaires sont ajoutés pour transformer les ingrédients (enzymes par exemple).

\section{Les produits}

\section{alimentaires}

\section{intermédiaires}

Situés entre les ingrédients et les aliments formulés, les produits alimentaires intermédiaires (PAI) sont des mélanges de matières premières et d'ingrédients qui ont subi une première transformation industrielle et qui sont destinés à être incorporés dans la fabrication d'un produit alimentaire final plus complexe, souvent pour les métiers de restauration ou de pâtisserie. Ainsi il y a une orientation forte vers une cuisine d'assemblage, notamment dans les collectivités, ce qui permet un gain de temps, une plus grande régularité des produits et une meilleure mâ̂trise des risques bactériologiques. Cette orientation peut entraîner la perte de savoir-faire technique, la modification de certaines recettes traditionnelles et ainsi la standardisation des produits fabriqués.

La gamme des PAI est extrêmement large et peut être classée en différentes familles: les bases élaborées (praliné, fondant, nappages), les bases prêtes à l'emploi (coulis, sauce anglaise, entremets), les préparations pour pâtes (génoises, biscuits, cakes, pâtes brisée, feuilletée), les pâtes de base crues ou cuites (croissant, pâte feuilletée, fond de tarte, pâtes à chou), ainsi que les préparations déshydratées à reconstituer (poudres à flan).

La filière des ovoproduits, ou produits à base d'œufs (Figure 2), peut être prise comme exemple de la fonctionnalité des ingrédients qui en sont extraits ainsi que de l'émergence des PAI. L'œuf de poule est qualifié lui-même d'ingrédient polyfonctionnel car, outre sa valeur nutritionnelle, il peut remplir simultanément plusieurs fonctions technologiques dans un même aliment formulé. Ses propriétés émulsifiantes*, foisonnantes*, gélifiantes, épaississantes, colorantes et aromatiques en font un ingrédient de base universel de la cuisine domestique et de l'agroalimentaire. Le blanc d'œuf est une référence en termes de foisonnement alors que le jaune d'œuf est l'agent émulsifiant par

\section{Références bibliographiques}

- Della Valle G. et al., La matrice alimentaire: définition, classification et caractérisation. In: Structure des aliments et effets nutritionnels, Fardet A. et al. éd. Quae, Paris, 2013.

- Anton M., Sirvente H., "La mayonnaise: une histoire d'assemblages", in Science culinaire - matière, procédés, dégustation, Lavelle C. éd. Belin, Paris, 2014.

- Anton M. et al., "Egg proteins», Handbook of Hydrocolloids ( $2^{\text {nd }}$ Édition), Phillips G.O., Williams P.A. éd. Woodhead, Abington (Cambridge), 2008.

- Nau F. et al., « De l'œuf aux ovoproduits », in Sciences des Aliments, Jeantet R. et al. éd., vol. 2. Lavoisier, Paris, 2007 : 107-38. 


\title{
2.7. La qualité des produits: les apports de la modélisation
}

\author{
Monique Axelos
}

\section{Les enjeux de}

\section{la qualité des produits}

Comprendre l'origine de la qualité* des produits alimentaires nécessite de comprendre à la fois l'impact de nombreux paramètres externes, aussi bien «au champ» que lors des procédés de fabrication, et des paramètres intrinsèques à l'aliment comme sa composition et son organisation structurale qui résulte des interactions entre les molécules le composant.

L’enjeu pour maîtriser la qualité consiste à relier les critères qui caractérisent la qualité des aliments aux données issues de leur composition et de leur structure. De nombreuses données expérimentales permettent d'établir certaines relations mais elles ne sont pas assez exhaustives pour permettre d'anticiper des changements de nos pratiques de productions et de transformations susceptibles d'accroître la durabilité de la chaîne alimentaire.

Face à une telle complexité, la modélisation s'avère être une approche pertinente et féconde. Une telle approche, si elle est courante dans de nombreux autres domaines comme l'automobile, la pharmacie ou la chimie, est encore très peu développée dans le domaine alimentaire. Derrière le terme de modélisation différents aspects sont abordés. Aux échelles nano et micrométriques il est possible de comprendre l'effet des interactions entre les molécules et de simuler les structures formées en fonction de leur nature et de paramètres tels que

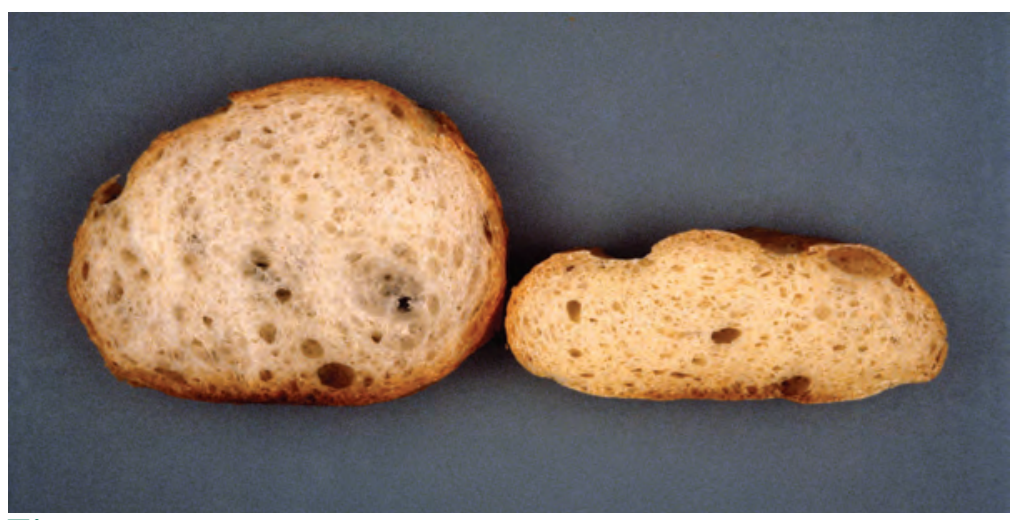

Fig. 1 - Panification. Étude des levures. Gonflement du pain. (Chantal Nicolas, INRA) la température, le $\mathrm{pH}$ ou la teneur en sels. À des échelles plus millimétriques il est possible de relier ces structures à des propriétés fonctionnelles. D'autres formes de modélisation permettent d'analyser les flux de matière et d'énergie et de simuler l'impact des procédés. D’autres enfin permettent de prendre en compte des savoir-faire ou des données qui traduisent l'appréciation des produits par le consommateur. L'intégration de ces différents modèles permet une approche dynamique de l'ensemble des processus pour aller vers des outils d'aide à la décision.

\section{Trois exemples de modélisation}

Trois exemples illustrent comment la modélisation peut apporter des clés pour comprendre l'origine des propriétés des aliments.

Le pain est le résultat de nombreuses étapes successives de transformation de la farine de blé. Derrière cette définition simple se cache en fait une succession d'opérations délicates à maîtriser qui mettent en œuvre tout un ensemble de savoirs et de savoir-faire partagés par des acteurs différents tout le long 


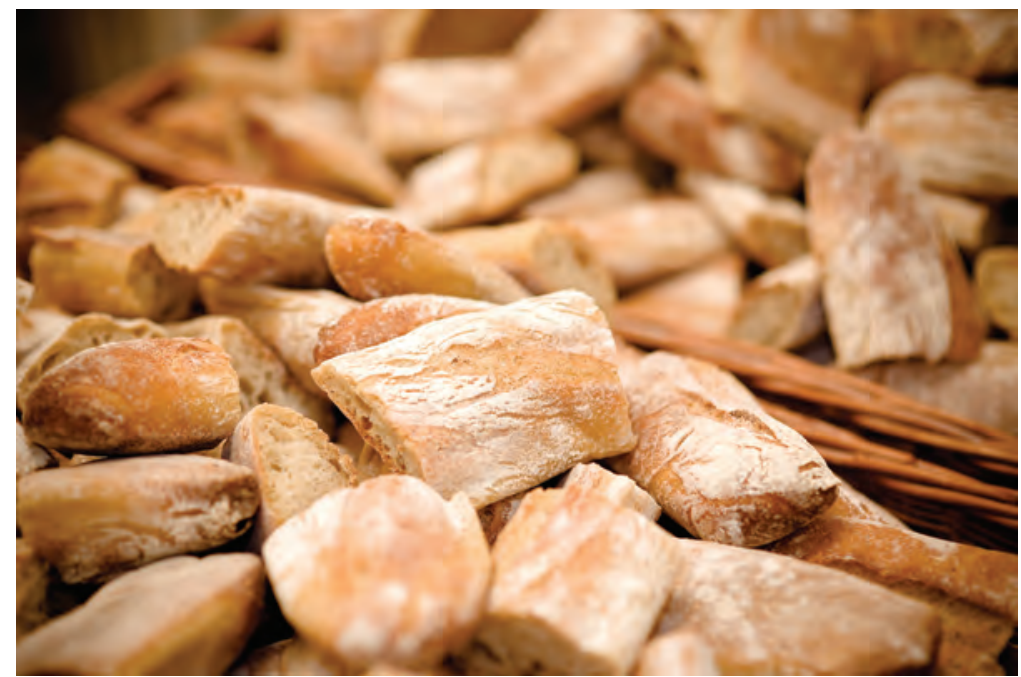

Fig. 2 - Baguettes. (Bertrand Nicolas, INRA)

de la filière, du semencier au boulanger en passant par le meunier. L'enjeu en terme de qualité est de permettre aux différents professionnels tout le long de la chaîne d'être en mesure de répondre aux cahiers des charges imposés par l'utilisateur suivant et cela jusqu'à la satisfaction du consommateur final. Un travail impressionnant a été réalisé par les chercheurs sur l'assemblage et la formalisation des connaissances en panification française qui a abouti à la réalisation d'une maquette informatique «Ascopain» permettant de capturer les savoir-faire, de confronter les expériences et de prédire l'incidence de la technologie de fabrication sur les caractéristiques finales du pain. Cette approche très novatrice d'assemblage des connaissances permet non seulement une sauvegarde des savoir-faire à connotation patrimoniale (la baguette française face au pain de mie international) mais aussi de bien identi- fier les spécificités de ces savoir-faire, ce qui est une garantie de qualité et de transparence pour le consommateur. De plus ces travaux contribuent à l'amélioration de la compréhension et de la communication entre tous les acteurs de la filière avec pour objectif de mettre en adéquation la farine aux conditions de travail du boulanger plutôt que l'inverse.

La texture des desserts lactés trouve son origine dans la structure des associations de protéines laitières qui sont présentes à la surface des gouttelettes de matière grasse du lait. Pour faire varier la texture des desserts, un modèle mathéma- tique a été construit qui permet de simuler les interactions entre les protéines présentes à la surface d'une gouttelette et entre les gouttelettes, et cela sur plusieurs échelles de taille. Ce modèle permet ainsi de prédire l'impact de la concentration en protéines, de la nature des protéines et de la température sur les propriétés d'écoulement des desserts, de très liquide à presque solide. Il permettra au final de proposer de nouvelles textures. Il peut être étendu à l'étude du remplacement des protéines animales par des protéines végétales.

Au-delà des produits eux-mêmes il est particulièrement important de comprendre leur impact sur l'homme. Dans le cadre de la réduction du contenu en sel dans les aliments qui est un problème majeur de santé publique, un modèle mécanistique a permis de relier la perception du goût salé à la libération effective du sel dans la salive, aux paramètres physiologiques de la mastication et à la fragmentation des aliments en bouche c'est-à-dire à leur structure et leur composition. Ce type de modèle permettra de formuler des aliments répondant à des critères à la fois nutritionnels (réduction effective du sel), et organoleptiques* (maintien du plaisir à consommer).

\section{Références bibliographiques}

- Chiron et al., Démarche de formulation des farines de blé appliquée à la panification. In: Science culinaire-matière, procédés, dégustation, Lavelle C. éd. Belin, Paris, 2014.

- Fardet A et al., Structure des aliments et effets nutritionnels, Quae, Paris, 2013. 


\subsection{Concilier qualité nutritionnelle et qualité sensorielle des aliments}

\section{Elisabeth Guichard}

À travers les dispositifs mis en place dans le cadre des Programmes Nationaux Nutrition Santé (PNNS*), les pouvoirs publics français ont choisi d'inciter à l'amélioration de la qualité nutritionnelle des aliments. Ces mesures impliquent une modification de la composition des aliments afin d'éviter la consommation excessive de sel, sucre et de matières grasses par exemple, qui peuvent contribuer au développement de certaines pathologies (hypertension, risques cardiovasculaires, diabète, obésité...). Les industriels sont ainsi amenés à reformuler leurs produits tout en maintenant une bonne acceptabilité pour le consommateur. Or, il ressort de l'expertise scientifique sur les comportements alimentaires coordonnée par Patrick Etiévant que le goût joue un rôle déterminant sur les choix alimentaires des consommateurs et donc sur leur santé.

\section{Les mécanismes}

La première étape de transformation de l'aliment lors du processus complet de digestion se situe dans la bouche. Sous l'action conjointe de la salive et de la mastication, l'aliment est déstructuré et les molécules responsables du goût sont libérées. Pour atteindre les récepteurs olfactifs et être perçues, les molécules responsables de l'arôme sont transférées de la cavité buccale vers la cavité nasale (Figure 1). La nature des constituants de l'aliment et leur organisation modulent l'accessibilité de ces molécules à nos récepteurs sensoriels.

Les mécanismes mis en jeu de la mise en bouche de l'aliment jusqu'à la perception sont complexes. De manière générale, les molécules responsables de l'arôme sont plus solubles dans la matière grasse que dans l'eau. Les aliments moins riches en matière grasse libèrent ainsi les arômes plus vite, induisant une perception rapide et moins persistante. Les protéines étant plus ou moins susceptibles d'interagir avec les molécules de la flaveur (ensemble des sensations olfactives, gustatives et tactiles), une modification de la source protéique conduit à des modifications de libération de ces molécules. Les agents de texture ajoutés dans les aliments reformulés sont susceptibles de réduire la libération des molécules d'arôme par des interactions plus ou moins spécifiques (exemple des complexes entre arômes et amidon) ou par la formation d'un réseau qui limite leur diffusion. Des modifications de composition en ingrédients peuvent également conduire à l'apparition de défauts d'arôme, comme les réactions d'oxydation de la matière grasse ou les odeurs résiduelles de certaines fractions protéiques. De plus, la connaissance des effets individuels des différents ingrédients entrant dans la composition d'un aliment ne suffit pas à prédire la libération des molécules de la flaveur et leur perception. En effet l'organisation des différents ingrédients structure l'aliment pour lui conférer des propriétés de texture particulières. Ainsi, la densité du réseau protéique dans des produits laitiers a un effet sur la mobilité du sel et sur sa libération en bouche: un réseau plus dense limite la mobilité du sel et son transfert de l'aliment dans la salive, ce qui a pour effet de diminuer l'intensité de la perception salée.

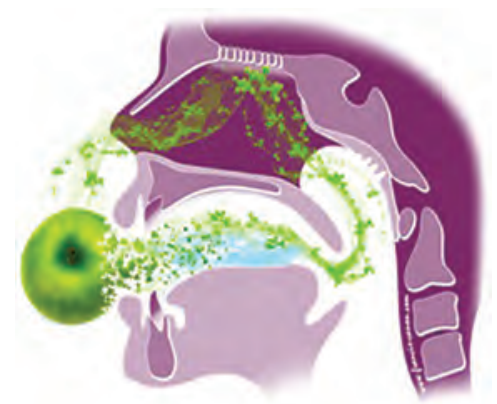

Fig. 1 - Libération des molécules responsables du goût lors de la mastication. 


\section{Perception des goûts}

La manière dont l'aliment est déstructuré en bouche varie en fonction de la physiologie de l'individu (composition de la salive, activité masticatoire). Une activité masticatoire intense augmente la libération des molécules de la flaveur et conduit à une perception accrue.

C'est au niveau du système nerveux central que convergent les informations transmises par les différents organes des sens. Lors de la consommation d'un aliment il y a une arrivée quasi simultanée des informations concernant les composantes de la flaveur et de la texture. L'intégration de ces différentes informations peut engendrer des interactions cognitives qui reposent sur des associations préalablement acquises. Par exemple l'ajout d'un arôme «fraise » renforce la perception de la saveur sucrée alors que l'ajout d'un arôme « sardine » renforce la perception de la saveur salée. De même, la saveur acide renforce la note odorante « citron » et diminue la note « vanille $»$. Des interactions perceptives ont été mises en évidence entre la perception du gras et celle du salé mais les effets dépendent à la fois de la teneur en sel et en matière grasse ainsi que de la structure de l'aliment, ce qui montre bien le niveau de complexité associé. Il est donc important non seulement de connaître le mode de libération des molécules de la flaveur* mais également de comprendre les méca-

\section{Références bibliographiques}

- Etiévant P., Les comportements alimentaires. Choix des consommateurs et politiques nutritionnelles, Quae, Paris, 2012.

- Etiévant P. et al., Les Comportements alimentaires. Quels en sont les déterminants? Quelles actions, pour quels effets? Expertise scientifique collective, INRA, Paris, 2010.

- Guichard E et al., Texture et flaveur des aliments, vers une conception maîtrisée, Educagri, Dijon, 2012. nismes sous-jacents à la perception des mélanges.

Une meilleure compréhension des effets d'une modification de composition des aliments sur la libération des molécules odorantes et sapides doit permettre l'élaboration de modèles mécanistiques prédictifs de cette libération en relation avec la perception. Ainsi, les industriels de l'agroalimentaire pourront bénéficier des outils nécessaires pour une reformulation des aliments, dans une démarche raisonnée et non plus empirique. 


\title{
2.9. Comment conserver les aliments: stérilisation, pasteurisation, surgélation, séchage, $4^{e}$ gamme
}

\author{
Catherine Renard
}

On conserve les aliments pour en préserver la comestibilité à long terme. Certains aliments se conservent d'eux-mêmes (fruits secs, grains, graines) mais la plupart demandent un traitement. La conservation doit assurer la sécurité microbiologique et prévenir la dégradation de l'aliment par les enzymes (endogènes ou liés à des microorganismes d'altération) et l'oxydation (Figure 1). Ces méthodes s'appliquent donc surtout aux aliments à haute teneur en eau, les plus favorables à la croissance des microorganismes ainsi qu' à l'expression des enzymes endogènes.

Les deux options consistent à éliminer les microorganismes ou à rendre les aliments et leur environnement impropres à leur croissance. Les méthodes inhibant la croissance des microorganismes sont le froid, la diminution de l'activité de l'eau* (séchage, ajout de sel, d'alcool ou de sucres), la compétition avec d'autres microorganismes (fermentations), l'utilisation d'agents chimiques (fumage, vinaigre, additifs). L'élimination des microorganismes doit être assortie de conditions de stoc- kage adaptées: emballage étanche, basse température.

Deux niveaux d'élimination des microorganismes peuvent être distingués : soit une pasteurisation au sens large, c'est-à-dire l'élimination des formes végétatives des microorganismes pathogènes et banaux, pour une durée de vie de quelques jours à quelques semaines avec conservation au froid et DLC* (date limite de consommation), soit une stérilisation c'est-à-dire l'élimination des formes végétatives et sporulées*, pour des produits capables de se conserver à température ambiante avec une DLUO* (date limite d'utilisation optimale) jusqu'à quelques années. Un facteur fondamental pour l'intensité des traitements est le $\mathrm{pH}$ de l'aliment: la majorité des bactéries, dont celles responsables des toxiinfections alimentaires

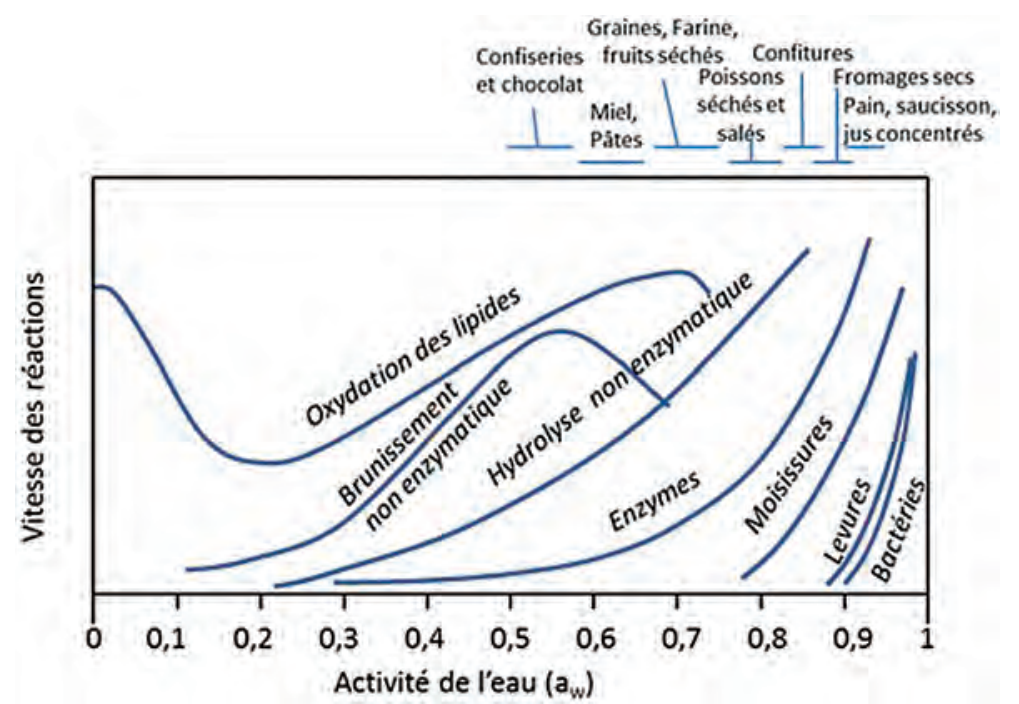

Fig. 1 - Activité de l'eau* et stabilité des aliments: vitesses des principales réactions de dégradation, quelques exemples d'activité de l'eau dans les aliments 
Tableau 1. L'intensité nécessaire des traitements de conservation en fonction de l'acidité des aliments

\begin{tabular}{|l|l|l|}
\cline { 2 - 3 } \multicolumn{1}{c|}{} & \multicolumn{1}{c|}{$\begin{array}{c}\text { Aliment acide } \\
(\mathrm{pH}<4.6)\end{array}$} & \multicolumn{1}{c|}{$\begin{array}{c}\text { Aliment peu acide } \\
(\mathrm{pH}>4,6)\end{array}$} \\
\hline $\begin{array}{l}\text { Pasteurisation } \\
\begin{array}{l}\text { Destruction des } \\
\text { formes végétatives } \\
\text { des microorganismes }\end{array}\end{array}$ & $\begin{array}{l}\text { Conservation } \\
\text { à température } \\
\text { ambiante, DLUO* }\end{array}$ & $\begin{array}{l}\text { Conservation } \\
\text { au froid, DLC }\end{array}$ \\
\hline $\begin{array}{l}\text { Stérilisation } \\
\begin{array}{l}\text { Destruction des formes } \\
\text { végétatives et sporulées }\end{array}\end{array}$ & $\begin{array}{l}\text { Conservation } \\
\text { à température } \\
\text { ambiante, DLUO* }\end{array}$ \\
\hline
\end{tabular}

et notamment du botulisme, les levures, les moisissures, ne poussent pas en milieu acide, et l'inactivation y est plus efficace. La limite est fixée à 4,6 (Codex Alimentarius*) : en dessous une pasteurisation est suffisante, au-dessus une stérilisation sera nécessaire (Tableau 1).

Le moyen le plus versatile de destruction des microorganismes étant la chaleur, ce traitement s'accompagne souvent d'une cuisson, qui, elle, a pour but de rendre les aliments palatables*. Les optima ne sont pas forcément les mêmes: la cuisson peut demander un chauffage plus (légumes secs) ou moins prononcé (haricots verts, fruits au sirop) que la conservation. La vitesse des réactions chimiques étant moins accélérée par l'augmentation de la température que l'inactivation des bactéries, en général les traitements à haute température et de courte durée préservent mieux les vitamines, pour un même niveau d'inactivation des microorganismes.

\section{Stérilisation}

\section{des aliments}

La stérilisation requiert des traitements thermiques à des températures supérieures à $100{ }^{\circ} \mathrm{C}$. L'appertisation est la principale méthode utilisée; elle a été initiée à partir des travaux de Nicolas Appert (1795). Elle consiste en un traitement thermique suffisant pour inactiver les formes végétatives et sporulées* (à l'exception de quelques bactéries thermorésistantes non responsables de toxiinfections alimentaires), appliqué à l'aliment dans son emballage hermétique final. La température de référence de l'appertisation est de $121,1{ }^{\circ} \mathrm{C}$, mais des températures de $110^{\circ} \mathrm{C}$ à $130{ }^{\circ} \mathrm{C}$ sont utilisées pour les aliments faiblement acides ( $\mathrm{pH}>4.6$ ). Outre les conserves classiques sous emballages rigides (boite en métal ou bocal), il s'est développé une gamme de produits sous emballage plastique souple. Ces emballages n'étant pas étanches à l'oxygène, les propriétés organoleptiques peuvent se dégrader, et les industriels préconisent des DLUO* plus courtes (de l'ordre de l'année et non de 2 à 5 ans).

Le traitement UHT (Ultra Haute Température, supérieure à $135^{\circ} \mathrm{C}$, pendant 1 à 2 s) inactive également les spores, permettant une conservation de quelques mois à température ambiante. Les temps très courts font qu'il n'est adapté qu'aux produits liquides (lait, depuis les années 1960) ou quasi liquides (soupes). Il est appliqué au produit avant emballage et exige un conditionnement aseptique. L'irradiation aux rayons gamma permet également la stérilisation des aliments mais est peu utilisée.

\section{Pasteurisation}

\section{et traitements proches}

La pasteurisation regroupe un ensemble de traitements thermiques moins prononcés que pour la stérilisation, à des températures inférieures à $100{ }^{\circ} \mathrm{C}$, qui diffèrent par leurs températures et durées (entre $62^{\circ} \mathrm{C}$ et $88^{\circ} \mathrm{C}$, typiquement $70-85^{\circ} \mathrm{C}$, $15 \mathrm{~s}$ à une minute). La «flashpasteurisation $\gg\left(1-2 \mathrm{~s}, 85-95^{\circ} \mathrm{C}\right)$ ou la pasteurisation courte à haute température s'appliquent aux produits liquides, et sont suivies d'un remplissage à chaud ou aseptique. Les produits «sous vide » ou les fruits et légumes de $5^{\mathrm{e}}$ gamme (tableau 2), sont emballés avant pasteurisation, ce qui implique des durées plus longues pour atteindre les températures 《à cour » de l'aliment. L'atmosphère appauvrie en oxygène dans l'emballage contribue à leur conservation. La pasteurisation est suffisante pour une conservation à température ambiante des aliments acides $(\mathrm{pH}<4,6)$. Pour les aliments peu acides, ces traitements exigent des conservations sous chaîne du froid, avec des DLC* de quelques jours ou quelques semaines. Leur commercialisation ne s'est développée qu'à partir des années 1990.

La pascalisation consiste à appliquer à l'aliment (préemballé sous emballage souple) des pressions de l'ordre de quelques centaines de kilopascals (quelques milliers de bars). Le principe est connu depuis la fin du XIX ${ }^{e}$ siècle, mais les contraintes physiques (enceintes et pompes) puis réglementaires font qu'elle s'est développée en Europe seulement 
Tableau 2. Les différentes gammes de produits végétaux

\begin{tabular}{|c|c|c|}
\hline Désignation & Obtention & Durées et conditions typiques de stockage \\
\hline Première gamme & Produits frais & $\begin{array}{l}\text { Quelques jours (épinards) à quelques mois (carotte, } \\
\text { pomme), de } 0^{\circ} \mathrm{C} \text { à } 12^{\circ} \mathrm{C} \text { (selon le végétal) }\end{array}$ \\
\hline Deuxième gamme & Produits appertisés & Quelques années à température ambiante \\
\hline Troisième gamme & Produits surgelés & Quelques mois en froid négatif \\
\hline Quatrième gamme & Produits «frais découpés» & Quelques jours à quelques semaines au froid positif \\
\hline Cinquième gamme & Produits pasteurisés ou «sous vide» & Quelques semaines au froid positif \\
\hline Sixième gamme & Produits déshydratés & $\begin{array}{l}\text { Quelques mois ou années à température } \\
\text { ambiante, préservé de l'humidité }\end{array}$ \\
\hline
\end{tabular}

depuis les années 2000. Elle permet l'inactivation des formes végétatives tout en préservant les qualités nutritionnelles, les vitamines étant peu sensibles aux hautes pressions. Les enzymes endogènes ont une sensibilité intermédiaire et l'une des limites de la pascalisation est la possible activation (polyphénoloxydases* ${ }^{*}$ ou rémanence (pectineméthylestérases ${ }^{*}$ ) de certaines activités, notamment dans les produits végétaux, conduisant à des changements organoleptiques perceptibles. Les produits pascalisés, même acides, sont stockés au froid avec des DLC* de quelques semaines.

La thermisation (température inférieure à $62^{\circ} \mathrm{C}$ ), appliquée au lait, diminue seulement la charge bactérienne. Elle permet de préserver une partie de la flore lactique (pour une transformation en fromage par exemple).

\section{Conservation}

\section{par le froid négatif}

Le froid négatif permet des conservations de longue durée, par deux effets: d'une part l'«élimination » de l'eau liquide, transformée en glace, et d'autre part le ralentissement de la multiplication des microorganismes et des réactions.
La température de $-18{ }^{\circ} \mathrm{C}$ correspond à l'arrêt de toute multiplication, y compris des levures, mais certaines réactions, notamment d'oxydation, ont encore lieu à faible vitesse (lipides insaturés, caroténoïdes). Le transport de viande congelée depuis l'Argentine remonte aux années 1880 , mais le développement des produits congelés en France date des années 1970 avec l'apparition des congélateurs domestiques.

Surgelés et congelés se distinguent par la vitesse de congélation. La surgélation exige un refroidissement rapide: le produit est soumis à une température d'environ $-40{ }^{\circ} \mathrm{C}$ pour arriver rapidement à $-18^{\circ} \mathrm{C}$ à cœur. Elle s'applique industriellement à des produits de petite taille (épaisseur inférieure à $10 \mathrm{~cm}$ ) et produit de nombreux petits cristaux de glace, et donc préserve mieux l'intégrité cellulaire et la texture. La congélation consiste en un refroidissement lent et s'applique plus spécifiquement aux grosses pièces. Les cristaux de glace sont moins nombreux et plus gros, avec une altération marquée de l'intégrité cellulaire. Les procédés utilisent soit un contact avec des parois froides, soit des gaz froids (air glacé en lit fluidisé), soit des liquides cryogéniques (solutions de sel, sucre ou azote liquide, $\mathrm{CO} 2$ ).

Les microorganismes n'étant pas détruits par le froid, leur multiplication reprend à la décongélation. Il faut un maintien rigoureux

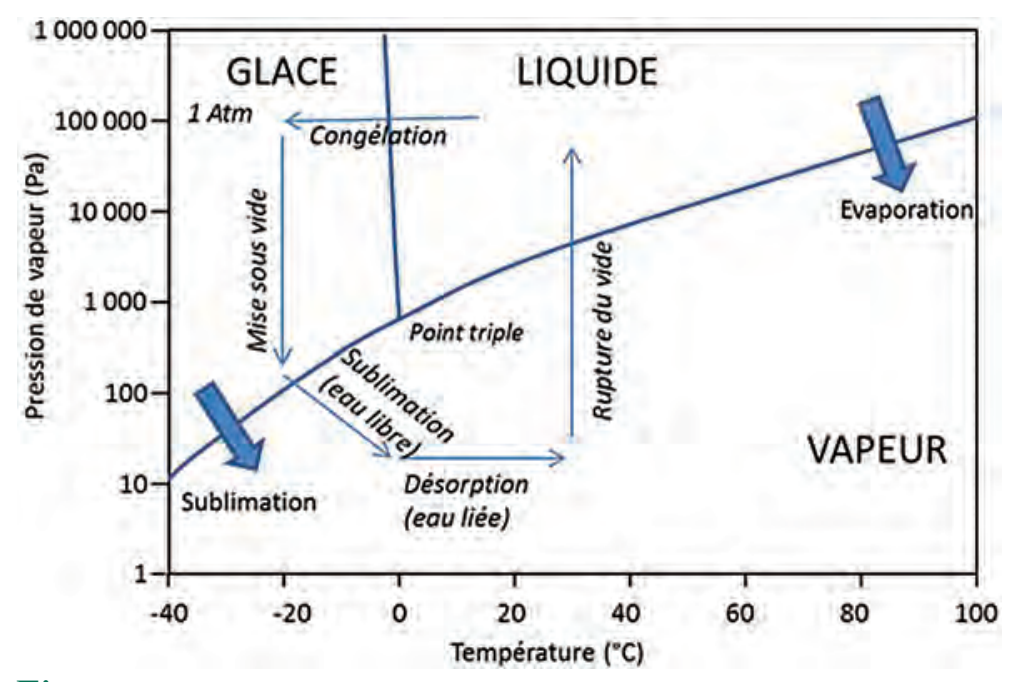

Fig. 2 - Diagramme de phase de l'eau et cycle de lyophilisation 
de la chaîne du froid (y compris par le consommateur), et la décongélation doit être rapide. Les petites pièces peuvent être cuites directement, limitant aussi les pertes par exsudation. Il faut décongeler complètement au préalable les grosses pièces, soit à $4{ }^{\circ} \mathrm{C}$ (plusieurs heures), soit au four microonde à faible puissance (quelques dizaines de minutes).

\section{Séchage}

La déshydratation permet à la fois de conserver et de diminuer l'encombrement des aliments. Elle peut être mise en jeu soit par évaporation de l'eau liquide (ébullition, entraînement*), soit par sublimation de la glace (lyophilisation). Le plus courant (et le moins coûteux) est le séchage par entraînement, où l'aliment est mis en contact d'un air chaud et sec (atomisation, lit fluidisé, tunnel de séchage). Les séchoirs sur cylindre sont utilisés pour déshydrater des aliments liquides ou pâteux : les cylindres chauffés intérieurement amènent le produit à sa température d'ébullition, une pellicule se forme en surface qui est rapidement desséchée et les flocons sont récupérés par raclage. La lyophilisation (Figure 2) permet une déshydratation du produit solide et à faible température, et donc la meilleure conservation des propriétés mais à un coût énergétique élevé. Le produit congelé est mis dans des conditions de température faible et de pression basse (inférieures au point triple de l'eau), et les vapeurs d'eau sont entraînées vers un piège froid pour leur condensation. La durée de lyophilisation dépend étroitement de l'épaisseur du produit. Les produits lyophilisés se réhydratent très facilement, ce qui peut être un avantage (dissolution) ou un inconvénient, notamment pour les produits riches en sucres qui doivent être emballés hermétiquement car ils ont une très forte propension à capter l'humidité de l'air et à s'agglomérer.

\section{Produits «frais découpés» \\ ou $4^{\mathrm{e}}$ gamme}

Les produits végétaux dits de $4^{\mathrm{e}}$ gamme se sont développés rapidement à partir des années 1980 (salades en sachet). Ce n'est pas à proprement parler une méthode de conservation, puisqu'elle diminue la durée de vie et qu'elle garde intacts les mécanismes enzymatiques et physiologiques. Les végétaux nettoyés sont découpés, lavés, emballés et stockés au froid $\left(4^{\circ} \mathrm{C}\right)$. L'atmosphère interne du sachet peut être modifiée (emballage sous atmosphère modifiée ou effet de la respiration). En effet, diminution de l'oxygène et accumulation du $\mathrm{CO} 2$ ralentissent le métabolisme des végétaux et donc leur dégradation,

\section{Référence bibliographique}

- Jeantet R. et al., Sciences des aliments 1. Stabilisation biologique et physico-chimique, Lavoisier, Paris, 2006. et diminuent la prolifération de certains microorganismes. Il ne faut pas éliminer totalement l'oxygène : cela conduirait à l'altération du végétal avec fermentation et risque de botulisme. La perméabilité des films est donc un critère important qui doit être adapté à chaque végétal.

Lors du lavage, des auxiliaires technologiques inactivent les microorganismes et rendent la surface de découpe moins favorable à leur croissance. Ce sont des composés oxydants (dérivés chlorés, acide peracétique*, ozone) ou acides (acide citrique ou peracétique); un antioxydant (souvent l'acide ascorbique) peut être ajouté pour limiter le brunissement enzymatique. Certains produits, particulièrement sensibles, nécessitent la présence d'une solution (d'acides citrique et ascorbique) pour limiter ce brunissement.

Depuis les années 1950 et grâce à la généralisation des chaînes du froid, se sont développées de nouvelles méthodes de conservation des aliments, qui permettent de mieux en préserver les caractéristiques intrinsèques, organoleptiques et nutritionnelles. Le défi qui est maintenant posé aux industries alimentaires est de pouvoir conserver les progrès faits en termes de qualité et de disponibilité tout en diminuant les coûts environnementaux des chaînes du froid. 


\subsection{Conserver par des méthodes traditionnelles: séchage, fumage, salage}

Isabelle Bianquis

Les techniques de séchage, salage et fumage, qui peuvent se combiner, sont destinées à la conservation et au stockage de denrées en vue de différer leur consommation pour des questions d'ordre gustatif, pratique ou rituel: assurer l'alimentation en période critique, se déplacer (sociétés nomades), échanger.

Le séchage constitue probablement la technique la plus ancienne et la plus pratiquée dans le monde. Poissons, viandes, céréales, végétaux, fruits, laitages font l'objet de ce type de conservation sèche soit à l'air libre, soit en local clos sous l'action de la chaleur.

L'eau étant au centre des problèmes de conservation, l'homme,

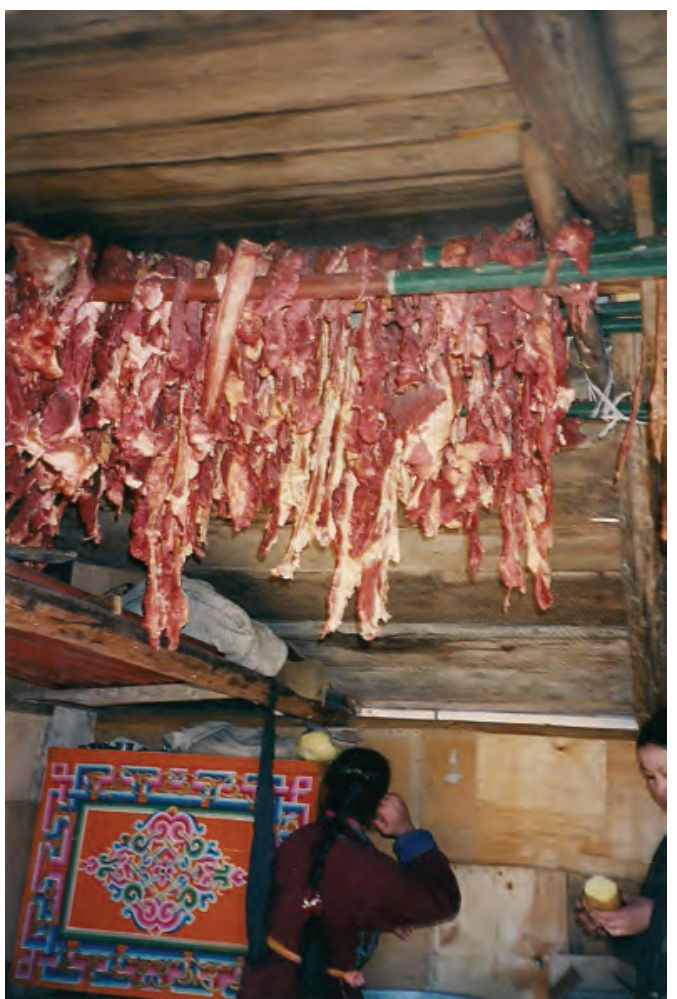

Fig. 1 - Viande séchée conservée sur un balcon d'appartement dans un quartier nord d'Oulan-Bator (photo Bianquis I, 2008).

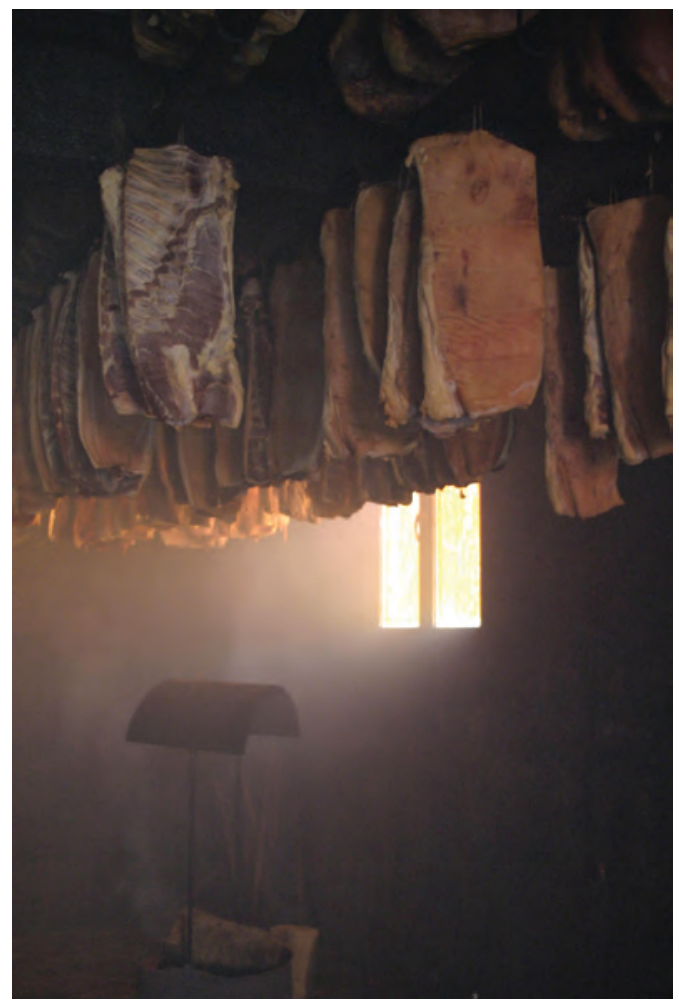

Fig. 2 - Fumage de viande au Njeguši, au Monténégro (photo Ralf Smallkaa). 
dans les milieux écologiques pourvoyeurs de sel, a découvert les vertus de ce dernier. La salaison consiste à recouvrir l'aliment de sel ou à l'immerger dans une préparation salée (saumurage). Dans un grand nombre de sociétés, l'utilisation du sel intervient plus dans les processus techniques que dans l'alimentation.

Très tôt, peut-être dès la fin du Paléolithique supérieur, l'homme constate que la viande ou le poisson exposé à la fumée se conserve bien. La technique de la fumaison dépend $\mathrm{du}$ milieu (avoir à disposition du combustible; pour cette raison, on la trouve très peu chez les Esquimaux), des saisons (on fait sécher et fumer en été et en automne dans les sociétés arctiques pour une consommation hivernale). Le boucanage, pratiqué notamment dans les Caraïbes, consiste à rôtir et fumer simultanément la viande déposée sur une claie (boucan).

Outre le séchage, le salage ou le fumage, on trouve, selon les sociétés et les milieux, d'autres pratiques de conservation : congélation naturelle, torréfaction, ébullition, fermentation.

L'anthropologue Marcel Mauss voyait dans la technique un «acte traditionnel efficace $\gg$ et pointait la relation dialectique entre les

\section{Références bibliographiques}

- Latour B., Lemonnier P., De la Préhistoire aux missiles balistiques. L'intelligence sociale des techniques, La Découverte, Paris, 1994.

- Leroi-Gourhan A., Milieu et techniques, Albin Michel, Paris, 1992.

- Ingold T., "The significance of storage in hunting societies", Les techniques de conservation des grains à long terme, Gast M. et al. éd. Éditions du CNRS, Paris, 1985 : 33-46.

techniques et le social. Les premières ne sont pas seulement une manière d'agir sur la matière, elles sont imbriquées dans le social et revêtent une fonction symbolique. Ceci est particulièrement vrai pour les sociétés dites traditionnelles dans lesquelles la plupart des membres sont concernés par l'activité technique.

Ces techniques ne peuvent s'étudier sans évoquer la pratique du stockage et la consommation. Il faut différencier le stockage économique et le stockage social, le premier permettant de disposer de biens de manière différée, le second, destiné au partage, s'inscrivant dans une idéologie de la réciprocité. La consommation renvoie aussi à des classifications et à des hiérarchies différenciées. Ainsi, en Mongolie, les produits frais, perçus comme neufs, sont valorisés et offerts à ceux que l'on veut honorer. À l'inverse, les aliments séchés (usés par le temps) font l'objet d'une consommation familiale. 


\title{
2.11. Les aliments fermentés au cour des régimes alimentaires
}

\author{
Sylvie Lortal
}

50 à $400 \mathrm{~g} \mathrm{~d}$ 'aliments et boissons fermentées sont consommées par jour et par personne dans le monde, ce qui représente selon les pays de 5 à $40 \%$ de la prise alimentaire. Plus de 5000 aliments fermentés sont répertoriés et parmi eux des fleurons des cultures locales. La fermentation, via l'action des microorganismes sur des matières premières extrêmement variées, a sécurisé et diversifié notre alimentation depuis plus de 10000 ans. Issus d'une longue tradition, durables, d'une infinie diversité aromatique, et contenant des quantités élevées de microorganismes vivants, les aliments fermentés ont une place particulière au cœur de nos régimes alimentaires.

\section{Une longue histoire et une extraordinaire diversité}

Lors de la fermentation, les microorganismes transforment les matières premières, via des modifications biochimiques. Celles-ci limitent la croissance des germes indésirables entraînant un allongement de la conservation (sécurisation), et génèrent parallèlement des goûts, couleurs et textures variés (diversification). Parmi ces modifica- tions figurent la consommation des sucres, la production d'acides organiques, ainsi que la production de centaines de composés aromatiques. Un grand nombre de matières premières d'origine animale ou végétale peuvent être fermentées, : lait, viande, poisson, œufs, céréales (riz, maïs, blé, seigle, avoine, sorgho, mil), fruits et légumes (choux, olives, cornichons, soja, haricot, carottes, manioc), cacao ou thé, et dans le but particulier de générer des boissons alcoolisées : raisin, malt, orge, et canne à sucre - cette liste n'est pas exhaustive. Entre l'Europe, l'A frique, l'Inde, ou l'Asie, selon les ressources locales, la carte des matières premières fermentées diffère grandement.

En revanche, les produits finis sont toujours des aliments à forte valeur ajoutée culturelle et gustative: pain, fromages, yaourts, charcuterie, condiments, sauces aromatiques, choucroute et innombrables aliments végétaux fermentés en Asie sans oublier le vin et la bière, présents sur tous les continents. Sous l'appellation générique «fromage », «bière » ou «sauce de soja $»$, des centaines de variantes existent selon les pratiques locales avec une remarquable diversité organoleptique. Ainsi, l'homme a développé un grand savoir-faire empirique autour de la fermenta- tion, savoir qui fait partie du patrimoine alimentaire d'une région, et ce bien avant la reconnaissance par Pasteur en 1865 du rôle joué par les microorganismes. Par ailleurs, les aliments fermentés traditionnels sont durables par essence, issus de la conjonction des ressources locales et de la créativité de l'homme, et impliquant des procédés réalisables sans équipement particulier, et sans chaîne du froid. Enfin, quand les ressources naturelles sont peu diversifiées (comme le poisson chez les Inuits) ou produites de manière saisonnière, la fermentation a permis d'établir des régimes assez diversifiés pour permettre la survie dans des biotopes extrêmes, autre forme de durabilité.

\section{Les aliments}

\section{fermentés sont}

des produits «vivants»

La plupart des aliments fermentés contiennent des niveaux élevés de micro-organismes vivants, d'1 million à 10 milliards par gramme. Depuis Pasteur, des générations de microbiologistes ont exploré les espèces présentes, leur contribution à la qualité finale du produit, et constaté une grande diversité d'ac- 
teurs : bactéries, levures et champignons. Cet écosystème microbien* provient de la matière première, de l'environnement, des pratiques de fabrication et parfois d'un ajout empirique de levains. Il varie donc dans le temps.

La maîtrise de ces flux microbiens a toujours été un défi, qui devient abordable aujourd'hui avec les outils de métagénomique*. Au $\mathrm{Xx}^{\mathrm{e}}$ siècle, la volonté de rationnaliser à grande échelle la production de ces aliments et de garantir l'absence totale de pathogènes a entraîné l'avènement de levains dont la biodiversité est très réduite au regard des fabrications artisanales, appauvrissement dont on ignore encore les conséquences en termes de santé.

\section{Intérêt nutritionnel des aliments fermentés}

Les aliments fermentés contiennent une biomasse microbienne élevée. Si la maîtrise de ces écosystèmes microbiens* progresse, on sait peu de choses des effets pour l'homme de leur consomma- tion régulière. Dans une période de montée des pathologies liées à l'alimentation, d'augmentation des allergies, de fragilisation d'une partie de la population, la question mérite d'être posée.

Au regard des travaux actuels, la fermentation augmente la valeur nutritionnelle des aliments. En effet, les micro-organismes peuvent produire in situ des vitamines (B ou $\mathrm{K}$ ) ou des acides aminés essentiels, et inactiver des facteurs antinutritionnels (fractions non comestibles de l'aliment). Elle peut aussi augmenter la digestibilité du produit via une hydrolyse* partielle des sucres (c'est le cas du yaourt dont le lactose est prédigéré par les ferments lactiques), elle peut réduire l'allergénicité des protéines végétales par une hydrolyse partielle. Outre la transformation biochimique bénéfique de la matière première, d'autres pistes existent quant au bénéfice nutri- tionnel: quel est l'effet sur le système immunitaire et sa maturation de la consommation depuis le plus jeune âge d'aliments avec des niveaux élevés de micro-organismes vivants et diversifiés ? Cet écosystème alimentaire peut-il influencer même transitoirement la composition ou l'activité du microbiote* intestinal, dont on connait désormais les liens étroits avec la santé de l'homme?

Enfin, dans les pays en voie de développement, la part des produits fermentés dans la sécurisation de l'alimentation (par la préservation des matières premières) et dans l'apport de micronutriments n'a pas été explorée à la mesure des enjeux de santé voire de survie des populations concernées. Sains, durables, sources d'innovation mais aussi accessibles aux plus pauvres, les aliments fermentés représentent une dimension essentielle dans l'alimentation humaine.

\section{Références bibliographiques}

- Tamang J.P., Kailasapathy K., Fermented food and beverages of the world, CRC Press, 2010.

- Zagorec M. et al., Ecosystèmes microbiens et préservation des aliments. CIAG La maîtrise du risque sanitaire dans l'alimentation humaine, INRA, 2012. 


\title{
2.12. Le rôle de la chaîne du froid: enjeux et défis actuels
}

\author{
Evelyne Derens-Bertheau et Onrawee Laguerre
}

\section{Le froid, enjeu capital}

La production alimentaire mondiale (5500 millions de tonnes) se compose d'un tiers environ de produits périssables (1800 millions de tonnes) qui pourraient être conservés sous régime de froid; or, seuls $22 \%$ le sont. L'importance d'une chaîne du froid efficace joue clairement un rôle déterminant dans la réduction des pertes. L'homme a observé, peut-être déjà 100000 ans avant J.-C., que le froid permettait de conserver les aliments plus longtemps. La glace naturelle a été stockée et utilisée très longtemps en complément des pratiques traditionnelles telles que le salage, le fumage. À la fin du XIX ${ }^{e}$ siècle, le développement du froid industriel a remplacé cette source naturelle et permis de développer la consommation de produits périssables dans des lieux éloignés de la production,

En effet, le froid ralentit d'une part le développement des microorganismes et, d'autre part, les réactions chimiques à l'origine de la dégradation des caractéristiques sensorielles et nutritionnelles. Pour une bonne conservation de l'aliment, il faut un produit sain, une réfrigéra-

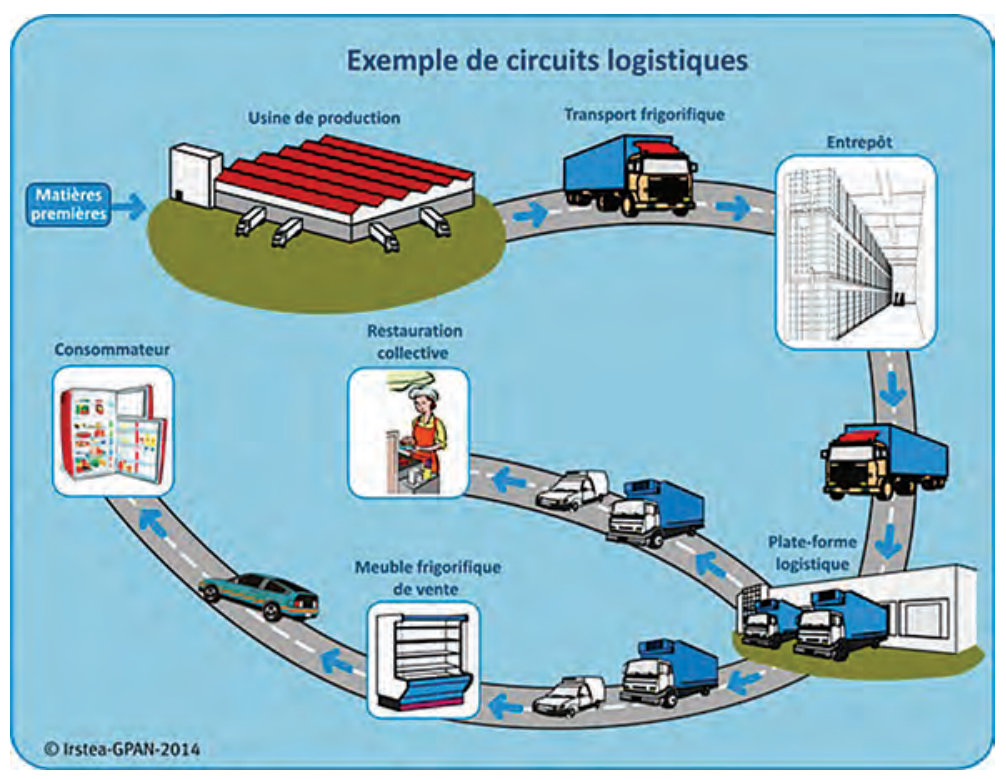

Fig. 1 - Un exemple de chaîne du froid. tion précoce et un froid continu; c'est ce dernier critère qui conduit à la notion de « chaîne du froid ».

On distingue deux catégories de produits alimentaires à conserver sous température dirigée : les produits surgelés à conserver à $-18^{\circ} \mathrm{C}$ ou en-dessous, et les produits réfrigérés à conserver selon les produits entre $0{ }^{\circ} \mathrm{C}$ et $+8^{\circ} \mathrm{C}$. En France, on consomme environ $36 \mathrm{~kg}$ d'aliments surgelés par an et par habitant, et $300 \mathrm{~kg}$ d'aliments réfrigérés en considérant les fruits et légumes.

\section{La chaîne du froid}

La chaîne du froid est une succession d'opérations qui s'effectuent sous température dirigée au moyen d'équipements frigorifiques, de la récolte ou de la fabrication du produit jusqu'à sa consommation (Figure 1). Elle utilise donc des entrepôts frigorifiques, des transports frigorifiques, des plates-formes logistiques, des meubles frigorifiques de vente et des réfrigérateurs ménagers. Dans le cas de la restauration collective, on a, après les platesformes, un stockage en chambre froide avant utilisation. Toutefois il n'y a pas une chaîne logistique type, mais une multitude d'enchaînements de ces différents maillons. $\mathrm{Si}$ 
Fig. 2 - Pourcentage estimé de produits «à risque » (jambon en tranches) en fonction de la durée de conservation dans un réfrigérateur. Courbe rouge: produit conservé dans la zone chaude du réfrigérateur, Courbe bleue: produit conservé dans la zone froide (d'après S. Duret, Modélisation des variabilités des transferts thermiques et hydriques dans la chaîne du froid pour la maîtrise de la qualité et de la sécurité sanitaire, Thèse, École Doctorale ABIES, 2013).

le transfert des produits d'une étape à l'autre entraîne des ruptures de la chaîne logistique, l'essentiel est de maintenir le produit du début à la fin en dessous de la température de conservation recommandée.

Les températures des produits les plus élevées se mesurent dans les meubles frigorifiques de vente et les réfrigérateurs domestiques. En France, la grande distribution s'est engagée à équiper les magasins de meubles frigorifiques de vente fermés pour les produits réfrigérés avant 2020. Cette mesure a été prise essentiellement pour des raisons d'économie d'énergie, mais elle aura probablement un impact fort sur le maintien de la température des produits. Déjà, une étude européenne réalisée en 2013 constate que les températures sont plus maîtrisées qu'il y a 10 ans en meuble frigorifique de vente ouverts; cette amélioration ne sera donc qu'amplifiée. Le maillon le plus faible reste donc maintenant celui du réfrigérateur domestique.

\section{Le rôle du}

\section{consommateur dans la chaîne du froid}

Lorsque le consommateur achète un produit et s'il ne le consomme pas immédiatement, il doit main-

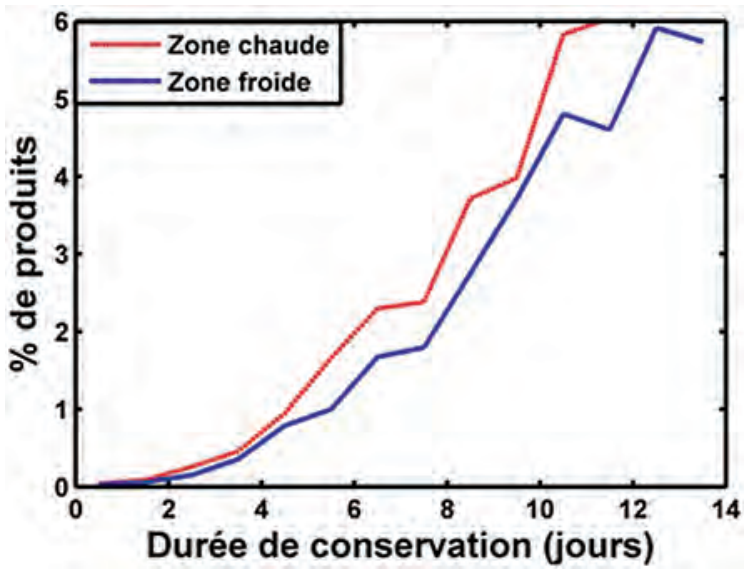
date limite de consommation.

Si les produits restent en moyenne 3 à 4 jours en réfrigérateur, ils peuvent parfois y rester 15 jours. Et si la température de conservation est au-dessus des $4{ }^{\circ} \mathrm{C}$ recommandés pour les produits très périssables, notamment pour les plats préparés réfrigérés, les micro-organismes peuvent s'y développer plus rapidement entraînant une possible intoxication alimentaire. La figure 2 montre le pourcentage estimé de produits dits «à risque » (ici paquets de jambon) dans un réfrigérateur domestique en fonction de la durée de conservation.

Le maintien d'une température donnée implique forcément l'utilisation d'équipements frigorifiques. À partir des années 1990, l'interdiction de certains fluides frigorigènes* (CFC, HCFC) ayant un impact sur

la couche d'ozone*, et plus tard la restriction d'autres fluides contribuant à l'effet de serre*, ont entraîné le recours accru aux fluides naturels (ammoniac, CO2, propane...). Par ailleurs, des technologies ayant moins d'impact sur l'environnement ont été développées : confinement des installations, réduction de la charge en fluides, utilisation de fluides frigoporteurs*, optimisation $\mathrm{du}$ fonctionnement de l'installation frigorifique. Par exemple, pour limiter les risques de fuite de fluide dans l'environnement, on peut soit améliorer l'étanchéité de l'installation, soit diminuer la charge en fluide frigorigène en utilisant des échangeurs de chaleur à micro-canaux (diamètre $1 \mathrm{~mm}$ ) ou des fluides frigoporteurs qui transportent le froid au lieu d'utilisation.

\section{Références bibliographiques}

- «100 ans d'innovation dans le Froid», Revue générale du Froid, 2008.

- Maîtrise de la chaîne du froid des produits carnés réfrigérés, Cemagref-AFF, 1999.

- Thévenot R., Essai pour une histoire du froid artificiel dans le monde, Institut International du froid, 1978.

- Monvoisin A., La conservation par le froid des denrées périssables, Dunod, Paris, 1923. 


\subsection{L'emballage alimentaire}

\section{Nathalie Gontard}

Qu'il soit en plastique (le plus important en volume), en papiercarton (premier matériau en valeur), en verre, en bois, en métal ou en matériau composite, l'emballage est mis en œuvre à la fin de la chaîne de production pour protéger et commercialiser l'aliment. L'emballage est souvent considéré indépendamment de l'aliment alors qu'il en est l'allié indispensable et indissociable. Après avoir été célébré pour une intensification et une diversification considérables de ses formes et de ses utilisations (deux prix Nobel en 1963 sur la chimie des plastiques), l'emballage est sorti du cœur des préoccupations de notre société à la fin du $\mathrm{xx}^{\mathrm{e}}$ siècle, où il reste remarqué pour ses innovations esthétiques et de praticité. À l'aube du XXI ${ }^{e}$ siècle, l'emballage est accusé de contaminer l'aliment et de polluer notre environnement, les coupables désignés étant les nombreux et complexes plastiques issus de la chimie du pétrole. Pourtant l'emballage se caractérise aujourd'hui par des innovations qui en font un élément indispensable à la durabilité de notre chaîne alimentaire.

\section{Économiser}

\section{les ressources}

L’emballage répond à la nécessité, accrue durant la deuxième partie du $\mathrm{XX}^{\mathrm{e}}$ siècle, de transporter et de stocker de façon plus fiable des denrées périssables. En réduisant les pertes de denrées alimentaires, il contribue ainsi aux enjeux mondiaux de sécurité alimentaire. Avant de communiquer, tracer, transporter, commercialiser, l'emballage a pour fonction première de protéger et de stabiliser l'aliment. Il définit matériellement autour de l'aliment une atmosphère favorisant la préservation des qualités gustatives et nutritionnelles ainsi que de la sécurité sanitaire des aliments, en protégeant ceux-ci des contaminations extérieures (poussières, micro-organismes, substances chimiques). On peut aussi priver l'atmosphère intra-emballage des réactifs entraî- nant la dégradation des aliments ou introduire des inhibiteurs de ces réactions. Les absorbeurs d'oxygène sont ainsi couramment utilisés pour réduire, sans additif ni traitement, les réactions d'oxydation des vitamines, des acides gras essentiels ou de composés aromatiques mais aussi le développement microbien. De nombreux autres emballages actifs* sont aujourd'hui commercialisés (absorbeurs d'eau, émetteurs de $\mathrm{CO}_{2}$, emballages anti-microbiens, etc.), et d'importants investissements en recherche y sont consacrés.

D’autres enjeux liés à la réduction des pertes alimentaires se jouent actuellement dans le champ

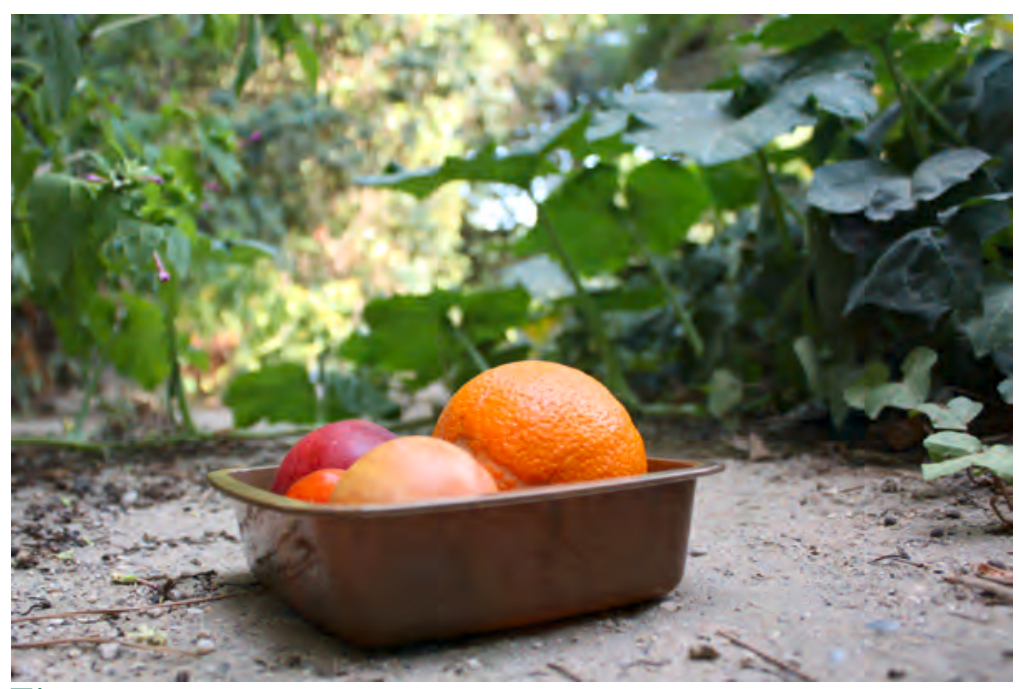

Fig. 1 - Barquette biodégradable, adaptée à la conservation de fruits et légumes frais, issue de sous produits des industries agro-alimentaires: mélange de PHBV issu de la fermentation d'effluents liquides des industries laitières, et de fibres lignocellulosiques issues du broyage de pailles de blé. Projet européen EcoBioCAP (www.ecobiocap.eu). (Photo Guilbert E, Montpellier) 
de l'emballage dit «intelligent »* qui informe tous les acteurs de la chaîne, consommateurs inclus, sur le suivi en temps et conditions réelles des qualités du produit. Il contribue ainsi à limiter les pertes liées aux marges de sécurité sanitaire sur la durée de conservation et aux mauvaises compréhensions des dates limites ou optimales de consommation. Des étiquettes intelligentes permettent par exemple de détecter la présence de bactéries pathogènes ou d'informer le consommateur de la qualité d'un aliment (comme l'état de maturité d'un fruit) sans avoir à le toucher ou à le sentir.

Source importante d'innovation, les nanotechnologies trouveront $20 \%$ de leurs débouchés en 2015 dans le secteur de l'emballage qu'il soit conventionnel, actif ou intelligent.

\section{L'emballage écologique}

Tout au long de leur cycle de vie, les matériaux d'emballage consomment des ressources d'énergie, souvent non-renouvelables, génèrent des émissions atmosphériques, des déchets. Un vortex de déchets plastiques dans l'océan Pacifique nord, appelé $7^{\mathrm{e}}$ continent, s'étend sur une superficie 6 fois plus grande que la France. Des efforts considérables ont été réalisés depuis les années 2000 pour encourager la minimisation des ressources (emballages réduits au «juste nécessaire ») et le recyclage sous la forme d'énergie (incinération), de matériaux décontaminés (PET, c'est-à-dire Polytéréphtalate d'éthylène, recyclé) et de compost fertilisant (emballages biodégradés avec les déchets organiques). Le remplacement des matériaux pétrochimiques par des matériaux issus de ressources renouvelables et non alimentaires (comme les déchets des industries agro-alimentaires) constitue une avancée très attendue de la recherche. L'enjeu se situe aujourd'hui dans l'amélioration de la balance environnementale du système «aliment emballé », en réduisant l'impact négatif du matériau sur l'environnement tout en améliorant le rôle positif de l'emballage sur la réduction des pertes alimentaires qui impactent fortement notre environnement.

Ce rapide panorama de l'emballage donne un aperçu de la diversité des rôles qu'il est amené à jouer dans toutes les étapes du cycle de vie de l'aliment, et du levier majeur qu'il représente pour répondre au défi de durabilité de nos systèmes alimentaires. Une transition vers des approches novatrices plus globales associées à des outils de modélisation permettra de prendre en compte dès la conception des emballages toutes les contraintes, risques et opportunités à relever.

\section{Références bibliographiques}

- Gontard N. et al., «Food Packaging Applications of Biopolymerbased Films", Films and coatings from renewable resources - An applications perspective, Placket D. éd., John Wiley \& Sons, New York, 2010.

- Angellier-Coussy H. et al., «Role of packaging in a smorgasbord of action for sustainable food consumption ", Agrofood Ind. High-tech 2013; 24: $15-9$.

- Barthélémy E. et al., "Safety evaluation of mechanical recycling processes used to produce polyethylene terephthalate (PET) intended for food contact applications ", Food Add Contam A 2014; 31: 490-7. 


\title{
2.14. Les pratiques et techniques culinaires
}

\author{
Bruno Laurioux
}

Le rôle de la cuisine est central car elle transforme les aliments acquis en aliments consommables - c'est-à-dire à la fois digestibles, acceptables culturellement et agréables au goût. Pour cela, elle met en œuvre des chaînes opératoires de gestes et d'actions tout en s'appuyant sur des connaissances.

\section{La cuisine,}

\section{un ensemble}

\section{de techniques}

Le terme français cuisine recouvre, depuis au moins le XII siècle, à la fois le lieu où l'on prépare les aliments, le fait de les préparer et le résultat de cette préparation. Trois réalités distinctes pour lesquelles l'anglais peut utiliser respectivement les mots kitchen, cookery et cooking. Identifiée depuis l'Antiquité comme un art manuel (technè en grec), la cuisine est d'abord un ensemble de savoir-faire, qui se transmet dans des milieux déterminés (la famille, le métier) et, plus récemment, par des moyens de diffusion comme la presse ou la télévision.

Les techniques culinaires soumettent des matières premières - les aliments - à des transformations physiques et chimiques. Citons, sans chercher à être exhaustifs : l'osmose inverse*, qui préside à la confection des bouillons; la caramélisation et

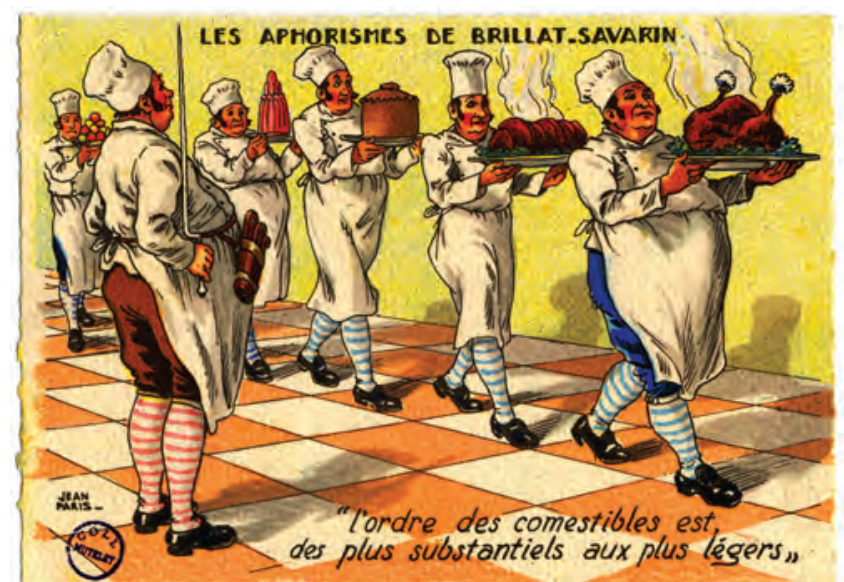

Fig. 1 - En avant-propos de son ouvrage Physiologie du goût, Brillat-savarin énonce vingt aphorismes. Ici l'aphorisme XI, illustré sur une carte postale ancienne.

la cristallisation dans le travail du sucre; le brunissement non enzymatique (la fameuse réaction de Maillard* qui donne, entre autres, sa couleur spécifique aux rôtis); l'émulsion*, dont le type même est la mayonnaise; enfin tous les phénomènes de durcissement, depuis la coagulation des protéines de l'œuf jusqu'à la gélification des pectines* dans la confiture. Ces transformations interviennent le plus souvent à l'issue d'une cuisson qui peut s'effectuer dans l'eau ou à la vapeur, dans une matière grasse (fritures et sautés), à chaleur sèche (rôtis et grillades) ou enfin selon des procédés mixtes (braisés et ragoûts).

La prise de conscience de la cuisine comme un ensemble de techniques jouant sur les caractéristiques physico-chimiques des aliments est ancienne. Les préfaciers des recueils de recettes qui, vers 1720-1730, prétendaient représenter une « nouvelle cuisine », caractérisaient celle-ci comme « une espèce de chimie ». L'auteur de la Physiologie du goût, Brillat-Savarin, est donc l'héritier d'une riche et longue tradition qui se poursuit au $\mathrm{XX}^{\mathrm{e}}$ siècle avec Édouard de Pomiane et sa Physique de la cuisine (où il propose de substituer 《 Gastrotechnie » à 《cuisine ») et jusqu'à aujourd'hui avec la gastronomie moléculaire, ses nouvelles méthodes (émulsification par ultra- 
sons) et ses produits fétiches, dont de nombreux additifs alimentaires.

\section{Des techniques}

\section{aux pratiques}

Décrire les techniques culinaires du point de vue physico-chimique ne suffit pas à en rendre compte. Elles prennent tout leur sens dans un contexte à la fois matériel, social et culturel qui explique leur diversité. On a pu ainsi distinguer la «grande cuisine » (telle qu'elle est pratiquée parmi les élites), qui vise à marquer fortement les différences sociales dans les sociétés hiérarchiques (de l'Égypte antique à l'Europe occidentale en passant par l'Islam et la Chine), des « petites cuisines » caractéristiques des sociétés dites hiératiques (par exemple en Afrique subsaharienne), c'est-à-dire à faible différenciation sociale. La diversité s'incarne aussi dans des systèmes culinaires spécifiques à une culture dont la terminologie rend compte. Dans la cuisine chinoise, le découpage est au moins aussi important que la cuisson proprement dite, selon le dicton «trois pour le fourneau, sept pour le billot »; la cuisson y est tournée vers la réalisation de consistances, de couleurs et d'arrangements visuels que traduisent plus d'une vingtaine de verbes différents.

Au-delà de ces diversités culturelles, les pratiques et techniques culinaires ont profondément évolué. Pour les périodes les plus anciennes, nous ne disposons que de vestiges archéologiques parfois fugaces: graines ayant subi une combustion qui peut résulter d'une cuisson (objet de la carpologie*); traces d'outils sur des os animaux trahissant une décarnisation et/ou une découpe (objet de l'archéozoologie*); résidus lipidiques encore présents sur les instruments de cuisson (depuis les pierres chauffées de la Préhistoire jusqu'aux céramiques médiévales). Seule la chimie analytique permet ici d'approcher le vaste domaine des matériaux organiques amorphes* (lait, huile, bière, vin).

À partir de l'Antiquité et surtout du Moyen Âge, des livres de cuisine, des inventaires notariés et de nombreuses représentations iconographiques permettent de compléter et d'interpréter les données des fouilles archéologiques. Ainsi peut-on repérer aux $\mathrm{XIV}^{\mathrm{e}}$ et $\mathrm{XV}^{\mathrm{e}}$ siècles le grand succès des préparations cuites à l'intérieur d'une pâte (nos pâtés en croûte) et la substitution aux grandes marmites (vouées aux grosses pièces bouillies) de pots de petite taille permettant de mijoter des portions réduites. Avec le XVII ${ }^{\mathrm{e}}$ siècle se met progressivement en place la gamme des fonds de sauces qui domineront la cuisine française jusque dans les années 1960. Parmi les tendances culinaires qui se sont affirmées récemment, on peut noter le retour aux cuissons à basse température - que le manque de temps des ménagères semblait avoir définitivement condamnées. On ignore en revanche la pérennité du four à micro-onde, dont l'invention, au sortir de la Deuxième Guerre mondiale semblait annoncer une véritable rupture par rapport aux modes de cuisson traditionnels.

\section{Références bibliographiques}

- Goody J., Cooking, cuisine and class: A study in comparative sociology, Cambridge University Press, 1982.

- Léry F., Technique de la cuisine, PUF, Paris, 1962.

- Regert M., Productions techniques et résidus alimentaires préhistoriques: les apports de la chimie organique, Technè 2001; 13-14: 71-7.

- Sabban F., Le système des cuissons dans la tradition culinaire chinoise, Annales Économies, Sociétés, Civilisations 1983; 38: 341-68. 


\subsection{Le cru et le cuit}

Jean-Pierre Williot

En ouverture du premier volume des Mythologiques, le grand ethnologue Claude Lévi-Strauss posait que «des catégories empiriques comme celles de cru et de cuit, mais aussi de frais et de pourri, de mouillé et de brûlé, pouvaient servir d'outils conceptuels pour dégager des notions abstraites $\gg$. Le titre de l'ouvrage, Le Cru et le Cuit, reflète la frontière entre deux états de la matière alimentaire autant qu'il contribua à fixer les principes du structuralisme. Il ne va pas malgré tout jusqu'à induire une présentation de tous les niveaux de cuisson. Pourtant l'évolution plurimillénaire des techniques de cuisson a engendré des élaborations culinaires complexes et des choix de consommation dont l'arbitrage résulte de goûts personnels, de règles religieuses, d'usages alimentaires ou de précautions liés à des savoirs intuitifs ou hygiéniques. La césure culturelle et technique qui oppose le mangeur de viande crue au mangeur de viande cuite recouvre des déclinaisons beaucoup plus nombreuses que celles qu'imaginait Lévi-Strauss.

La consommation de produits crus comprend plusieurs niveaux. Certains aliments entrent dans des registres alimentaires privilégiant leur dégustation sans cuisson, mets de luxe (caviar), denrée fraîche primeur goûteuse (fraise) produit conservé (tomate séchée). D'autres correspondent à des modes culinaires caractéristiques d'une pratique locale diffusée hors de leur aire initiale (comme c'est le cas des poissons crus de la cuisine japonaise en Europe occidentale depuis une quinzaine d'années) ou à des adaptations gastronomiques récentes dont la vogue est relayée des restaurants aux chaînes de distribution (carpaccio, préparations de viandes crues). En reliant le crudivorisme*, apparu dans les médias au cours de la dernière décennie, aux pratiques végétariennes inégalement diffusées dans le monde, de

\section{Références bibliographiques}

- Lévi-Strauss C., Mythologiques. Le cru et le cuit, Plon, Paris, 1964.

- Williot J.-P. (dir.), Du feu originel aux nouvelles cuissons. Pratiques, techniques, rôles sociaux du faire cuire, Peter Lang, Bruxelles, 2014.
l'Inde majoritaire à l'Europe occidentale minoritaire, mais connues dès l'Antiquité comme choix alimentaire, le cru tient une place spécifique.

Le passage aux préparations cuites introduit un autre registre gastronomique et une complexité des techniques de cuissons qui accompagne l'inventivité humaine dans toutes les aires culturelles. La diversité des techniques renvoie à l'énergie disponible. Le four polynésien permet de cuire à l'étouffée dans un trou du sol et limite la dépense de bois. Les choix de briques réfractaires et de bons combustibles introduisent dès l'Antiquité un savoir technique de la cuisson. L'emploi du charbon de terre (rare avant le XIX ${ }^{e}$ siècle), puis du gaz (vers les années 1890) et de l'électricité (surtout après la Seconde Guerre mondiale) fait place aujourd'hui à des gammes de possibilités nombreuses et précises (induction, microondes, basse température, sous vide). Marqueur culturel, la cuisson atteste par les vocabulaires qu'elle recouvre l'infinie variété du savoir-faire culinaire et des arômes qu'elle libère (bouillir, braiser, rissoler, mijoter, rôtir, marquer, griller, pocher). 


\title{
2.16. Les sauces au cœur de la cuisine française
}

\author{
Mary Hyman
}

Apparu au cours du XII ${ }^{\mathrm{e}}$ siècle, le mot salse, sause ou sausse prend très vite le sens général $d$ '« assaisonnement $\gg$. Mais jusqu' à la fin du $\mathrm{XVI}^{\mathrm{e}}$ siècle au moins, les cuisiniers doivent prendre en compte non seulement le goût mais aussi la nature ou «complexion » des aliments et assaisonnements, afin de garantir la bonne santé du mangeur. Depuis l'Antiquité, il régnait en médecine la théorie des humeurs selon laquelle l'univers était composé autour de quatre grands principes: le chaud, le froid, le sec et l'humide. La maladie survenait, croyait-on, lorsque le corps était en déséquilibre, d'où l'importance de composer des mets «tempérés $\gg$ : les animaux et végétaux qui étaient considérés comme froids et humides devaient être «corrigés » au moyen de sauces comportant une grande variété d'épices, toutes de nature plus ou moins chaude et sèche, et des acides, classés plus ou moins comme froids et secs. Si beaucoup d'aliments étaient cuisinés en sauce, donc « corrigés » au cours de leur cuisson, les viandes, volailles ou poissons pochés, rôtis ou grillés avaient besoin de sauces préparées et servies à part.

Vers la fin du Xvi ${ }^{\mathrm{e}}$ siècle les idées médicales et la façon de cuisiner commencent à changer. Les aliments sont alors considérés comme renfermant des principes

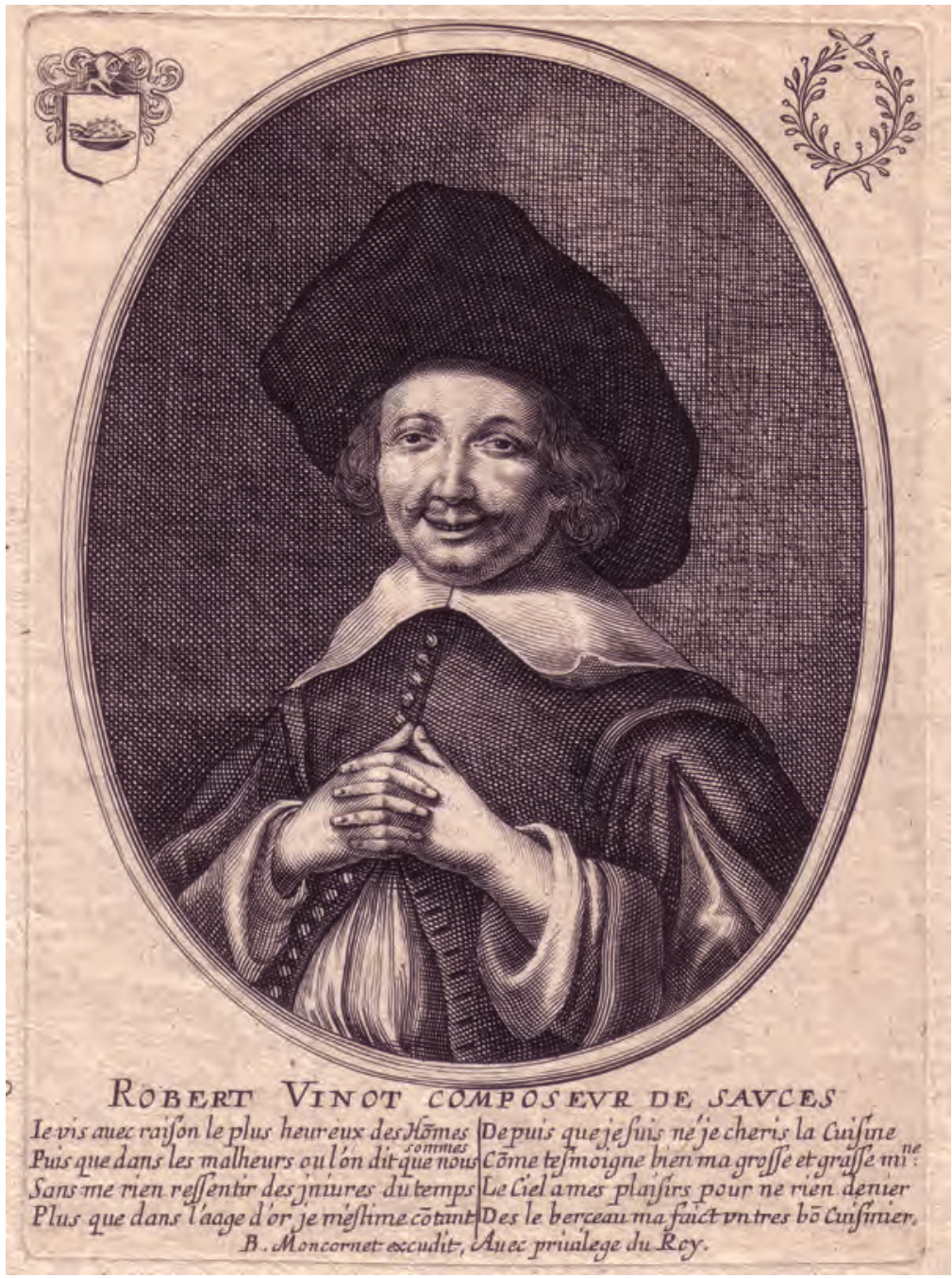

Fig. 1 - Portrait d'un cuisinier du xvII siècle. Robert Vinot, composeur de sauces. Texte modernisé: «Je vis avec raison le plus heureux des Hommes/ Puisque dans les malheurs où l'on dit que nous sommes/ Sans me rien ressentir des injures du temps/ Plus que dans l'âge d'or je m'estime content/ Depuis que je suis né je cheris la Cuisine/ Comme témoigne bien ma grosse et grasse mine : / Le Ciel à mes plaisirs pour ne rien dénier/ Dès le berceau m'a fait un très bon Cuisinier.» 
salés (qui donnent le goût), huileux (qui confèrent du moelleux et de la douceur), et spiritueux (responsables des odeurs). Ainsi, la plupart des épices et le sucre sont bannis des plats, assaisonnés désormais avec des herbes et condiments indigènes, afin de laisser transparaître le « vrai goût $\gg$ des choses. On privilégie les sauces au beurre et on prépare des « essences » et des « jus » goûteux et concentrés. Au XVIII ${ }^{\mathrm{e}}$ et XIX ${ }^{\mathrm{e}}$ siècles le nombre de sauces ne cesse de croître et celles-ci sont universellement reconnues comme la clé de voûte de la cuisine française.

En 1815, Antonin Carême les codifie en les divisant en « grandes » et «petites » sauces. Les premières, appelées également «sauces mères », étaient l'espa- gnole, le velouté, l'allemande et la béchamel (par la suite, furent également considérées comme sauces de base la hollandaise, la mayonnaise, et la vinaigrette). Il suffisait de leur ajouter d'autres ingrédients pour les diversifier en une multitude de «petites sauces »: une béchamel au fromage devenait ainsi une sauce Mornay, un velouté au fumet de poisson et à la crème était une sauce Normande.

Dès le début du $\mathrm{XX}^{\mathrm{e}}$ siècle des voix s'élèvent contre l'uniformi-

\section{Références bibliographiques}

- Hyman P., Hyman M., "Les associations de saveurs dans les livres de cuisine français du xvI siècle», Le Désir et le Goût: Une autre Histoire (XIII ${ }^{e}$-XVIII ${ }^{e}$ siècles), Redon 0. et al. éd. PUV, Saint-Denis, 2005: 135-50.

- Vitaux J., France B., Dictionnaire du gastronome, PUF, Paris, 2008. 


\subsection{Les épices}

\section{Paul Freedman}

Les épices sont des plantes aromatiques utilisées pour parfumer les aliments. Dans les langues d'Europe occidentale, elles se distinguent des herbes aromatiques par le fait qu'elles sont importées, habituellement séchées et piquantes, alors que les herbes aromatiques sont produites localement, vendues fraîches et n'ont pas une saveur brûlante.

L'usage des épices, en termes de quantités et de combinaisons entre produits, diffère considérablement entre les cuisines du monde et a évolué dans le temps. La nourriture indienne a été très transformée par l'introduction du piment d'Amérique, apporté par les Portugais au $\mathrm{XVI}^{\mathrm{e}}$ siècle. Auparavant, les assaisonnements indiens étaient plus âcres et poivrés mais moins brûlants qu'ils ne le sont aujourd'hui.

Sur le long terme, le goût européen pour les épices a d'abord évolué d'un engouement à une sévère limitation. Depuis l'Empire romain, la nourriture des élites sociales européennes était caractérisée par l'usage substantiel d'un grand nombre d'épices différentes. Le tournant culinaire commencé en France au XVII ${ }^{\mathrm{e}}$ siècle a réduit considérablement l'emploi des épices dans la cuisine. Les mets salés étant désormais séparés des mets sucrés, le sucre fut identifié au dessert et entraîna avec lui les épices (à l'exception du poivre). Ainsi, alors que la cannelle était employée par les recettes médiévales dans des sauces pour viandes et poissons, elle est réservée à la pâtisserie dans la plupart des livres de cuisine européens d'aujourd'hui.

À la fin du Moyen Âge, les épices les plus populaires étaient le poivre, le safran, le gingembre et la cannelle. Les épices provenant d'Indonésie orientale - clou de girofle, noix muscade et macis - jouissaient d'un prestige particulier et coûtaient bien plus cher que le poivre. Beaucoup d'épices inconnues de la cuisine européenne actuelle étaient régulièrement mentionnées dans les recettes médiévales: le galanga (commun en Thailande et en Indochine), le poivre long (épice très forte venue d'Inde) et ce qu'on appelait alors la «graine de paradis » (une

\section{Référence bibliographique}

- Freedman P., Out of the East: Spices and the Medieval Imagination, Yale University Press, New Haven, 2009. épice piquante originaire d'Afrique

Dans les dernières décennies, on a constaté un déplacement global des goûts occidentaux vers des saveurs plus éclectiques et plus épicées. Les parfums de l'Inde, du Mexique et du Moyen Âge sont désormais communs dans les villes européennes et pas simplement pour l'usage des communautés d'immigrants. Londres et New York sont bien connues pour accueillir une grande variété de cuisines importées et souvent épicées, alors que les traditions locales y sont faiblement représentées. Aux ÉtatsUnis et en Grande-Bretagne, le passage, depuis les années 1960, d'un goût très fade à une prédilection pour toutes les sortes d'assaisonnements piquants a été particulièrement marqué. Dans la haute cuisine et presque partout en Europe, ce phénomène se traduit par la volonté de créer une « cuisine fusion »* avec les goûts asiatiques, terrain favori d'expérimentation depuis la Nouvelle Cuisine des années 1970. occidentale). 